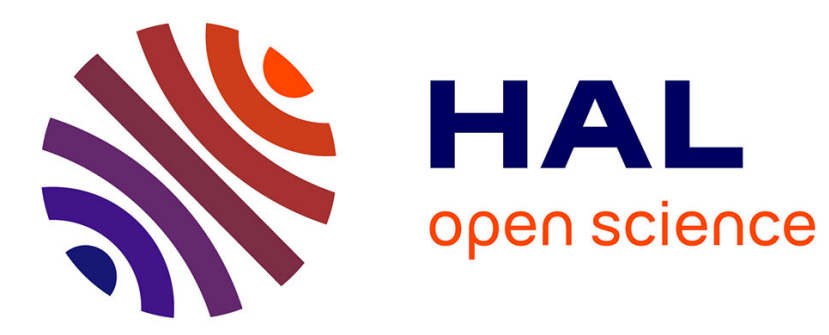

\title{
Dynamic building performance assessment using calibrated simulation
}

Jordan Brouns, Alexandre Nassiopoulos, Frédéric Bourquin, Karim Limam

\section{To cite this version:}

Jordan Brouns, Alexandre Nassiopoulos, Frédéric Bourquin, Karim Limam. Dynamic building performance assessment using calibrated simulation. Energy and Buildings, 2016, 122, pp.15. 10.1016/j.enbuild.2016.04.015 . hal-01354107

\section{HAL Id: hal-01354107 \\ https://inria.hal.science/hal-01354107}

Submitted on 17 Aug 2016

HAL is a multi-disciplinary open access archive for the deposit and dissemination of scientific research documents, whether they are published or not. The documents may come from teaching and research institutions in France or abroad, or from public or private research centers.
L'archive ouverte pluridisciplinaire HAL, est destinée au dépôt et à la diffusion de documents scientifiques de niveau recherche, publiés ou non, émanant des établissements d'enseignement et de recherche français ou étrangers, des laboratoires publics ou privés. 


\title{
Dynamic building performance assessment using calibrated simulation
}

\author{
Jordan Brouns ${ }^{\mathrm{a}, \mathrm{b}}$, Alexandre Nassiopoulos ${ }^{\mathrm{a}}$, Frédéric Bourquin ${ }^{\mathrm{c}}$, Karim \\ Limam $^{\mathrm{d}}$ \\ ${ }^{a}$ LUNAM Université, IFSTTAR, COSYS, SII, F-44340 Bouguenais, France \\ ${ }^{b}$ INRIA/IRISA, I4S Team, Campus de Beaulieu, 35042 Rennes cedex, France \\ ${ }^{c}$ Université Paris-Est, COSYS, IFSTTAR, F-77447 Marne-la-Vallée, France \\ ${ }^{d}$ LaSIE FRE-CNRS 3474, Université La Rochelle, La Rochelle, France
}

\begin{abstract}
Accurate building performance assessment is necessary for the design of efficient energy retrofit operations and to foster the development of energy performance contracts. An important barrier however is that simulation tools fail to accurately predict the actual energy consumption. We present a methodology combining thermal sensor output and inverse algorithms to determine the key parameters of a multizone thermal model. The method yields calibrated thermal models that are among the most detailed ones in the literature dealing with building thermal identification. We evaluate the accuracy of the resulting thermal model through the computation of the energy consumption and the reconstruction of the main energy flux. Our method enables one to reduce standard uncertainties in the thermal state and in the quantities of interest by more than 1 order of magnitude.
\end{abstract}

Keywords: building performance assessment, thermal diagnosis, inverse problem, parameter identification

\section{Introduction}

Energy retrofitting of the buildings stock is a major challenge to substantially reduce energy consumption in European countries. Energy performance contracts are a powerful tool to foster ambitious retrofit operations but their development is slowed down by the lack of accurate performance analysis tools. As a matter of fact, simulation tools used to assess the energy performance of existing buildings and design energy conservation measures lead to discrepancies between actual and computed energy performance, and thus fail to predict accurately the energy consumption after retrofit $[32,27,5,12]$. Techniques to

\footnotetext{
* Corresponding author

Email address: jordan.brouns@ifsttar.fr (Jordan Brouns)
} 
obtain calibrated simulation models are still needed and this has been an active research area in the two last decades $[1,2,29,28,16,37,26]$.

The literature on the topic often takes the direction of model simplification: in this approach, one looks for a sufficient prediction model that involves a few number of parameters that can be identified using a given set of measurement data. These methods are able to predict the overall thermal state, but lack insight into building parameters. They are particularly suitable for control problems. Many works on model reduction highlight the importance of the suitable choice of the model parameters [15, 18, 23].

The opposite direction consists in trying to correct the response of a detailed simulation model by adjusting some key parameters in the model. This leads to a problem that is usually more difficult to tackle because of the large number of parameters involved in a detailed simulation model compared to the available measured data.

Considering that a detailed simulation model is essential for the design of efficient energy retrofit measures, we address here the question of calibrating a simulation model based on standard dynamic multizone assumptions. The choice of such a detailed model enables to characterize all energy fluxes in the building under study, evaluate the true potential of refurbishment scenarios, and offers to the engineer in depth analysis of the building's behavior.

Model calibration can be treated in the context of inverse problems theory. The development of inverse problem approaches to building simulation goes back to the 1980s, [30]. Using this framework, we solve here a state-parameter identification problem which aims at determining sources and intrinsic properties of a mathematical model based on partial observations of the physical state, [3]. This kind of non-linear problems may be reformulated as optimization problems, where the unknowns are sought as minimizers of a cost-function evaluating the gap between the computed and the measured physical state, [34].

Special attention is given to assess the quality of the model that is obtained regarding two main criteria. The first is related to the ability of the model to accurately compute the energy needs in a building. The second one is related to its ability to compute the main energy fluxes that compose the thermal dynamical equilibrium of the building. We call quantities of interest the variables associated to these two criteria; they represent crucial information for the evaluation of refurbishment scenarios impact and return on investment. They are also a fundamental tool in the development of energy performance contracts.

The paper is organized as follows. First, we present the main modeling assumptions that are used and introduce a simple geometry that will be used for a case study. In the next section, we present the approach used for the resolution of the identification problem, based upon optimal control theory. The last section concerns various numerical tests that help evaluate the performance of the calibrated model under various situations with respect to the two abovementioned criteria. 


\section{Modeling assumptions and case study}

In this section, we first introduce the mathematical model used for this work. As explained before, it's a detailed building thermal model. We then present the case study and give mathematical definitions for the quantities of interest.

\subsection{Thermal model for building energy diagnosis}

The thermal model used in this work follows the standard multizone modeling assumptions: we consider homogeneous spatial distribution of the temperature field in the zones, and one-directional heat flux through walls, [4]. Multizone models directly derived from a continuous 3D formulation where fluids and solids are coupled by boundary layer exchange with Robin transmission conditions on interfaces, $[6,8]$.

The room air temperature is governed by an ordinary differential equation (ODE) evaluating the heat balance at the thermal node. Let $z \in \llbracket 1, N_{z} \rrbracket$ be the zone index, with $N_{z}$ the number of zones, and $T_{z}$ the corresponding air temperature. The heat balance equation writes:

$$
\left\{\begin{array}{l}
C_{z} \frac{d T_{z}}{d t}=\sum_{r=1}^{N_{z}} \mathcal{A}_{r z}+\overline{\mathcal{A}}_{z}+\sum_{s=1}^{N_{s}} S_{s}\left(\mathcal{C}_{s z}^{0}+\mathcal{C}_{s z}^{L}\right)+\mathcal{Q}_{z}+\mathcal{W}_{z}+\Phi_{z}^{b}+\Phi_{z}^{d} \\
T_{z}(t=0)=T_{z}^{0}
\end{array}\right.
$$

All terms are described in table 1, they account for solar gains, convective gains through walls (no matter the aeraulics) and air exchange, and internal gains from people and equipment. What we called convective gains actually account for both convection and conduction exchanges in boundary layers between zones and surfaces. We note $c_{a}$ the air heat capacity $\left(\mathrm{J} / \mathrm{K} \cdot \mathrm{m}^{3}\right), R_{z r}$ the airflow rate

\begin{tabular}{l|l} 
Flux & Definition \\
\hline $\mathcal{A}_{r z}=c_{a} R_{z r}\left(T_{r}-T_{z}\right)$ & interzone airmass exchange \\
\hline$\overline{\mathcal{A}}_{z}=c_{a} q_{z}\left(T_{a}-T_{z}\right)$ & airmass exchange with outside environment \\
\hline $\mathcal{C}_{s z}^{0}=h_{s z}^{0}\left(\theta_{s}(0, t)-T_{z}\right)$ & convection between surfaces and inside air \\
$\mathcal{C}_{s z}^{L}=h_{s z}^{L}\left(\theta_{s}\left(L_{s}, t\right)-T_{z}\right)$ & \\
\hline $\mathcal{Q}_{z}=\sum_{i} Q_{z}^{i}$ & internal gains from people and equipment \\
\hline $\mathcal{W}_{z}=\lambda_{z}\left(\Theta_{z}-T_{z}\right)$ & convective gains from heating devices \\
\hline$\Phi_{z}^{b}=A_{z} \tau_{z} \gamma_{z}^{b} \phi^{b}$ & gains from short-wave solar radiation \\
$\Phi_{z}^{d}=A_{z} \tau_{z} \gamma_{z}^{d} \phi^{d}$ & through windows
\end{tabular}

Table 1: Flux definition in the zones, with $\theta_{s}$ the wall temperature and $\Theta_{z}$ the heating device temperature.

between zones $z$ and $r\left(\mathrm{~m}^{3} / \mathrm{s}\right), q_{z}$ the air renewal rate $\left(\mathrm{m}^{3} / \mathrm{s}\right), T_{a}$ the mean outside air temperature $(\mathrm{K}), h_{s z}^{0}$ (resp. $h_{s z}^{L}$ ) the convective heat exchange coefficient between surface $s$ at surface $(x=0)$ (resp. $\left(x=L_{s}\right)$ ) and zone $z$ $\left(\mathrm{J} / \mathrm{K} \cdot \mathrm{m}^{2} . \mathrm{s}\right), \theta_{s}(x, t)$ the surface temperature $(\mathrm{K}), Q_{z}^{i}$ the internal gains from 
use $(\mathrm{J} / \mathrm{s}), \lambda_{z}$ the convective coupling between the heating device and the zone $(\mathrm{J} / \mathrm{K} . \mathrm{s}), \Theta_{z}$ the surface temperature of the heating device $(\mathrm{K}), A_{z}$ the windows area $\left(\mathrm{m}^{2}\right), \gamma_{z}^{b}$ (resp. $\gamma_{z}^{d}$ ) the beam (resp. diffuse) sun exposure coefficients for zone $z$, and $\phi^{b}$ (resp. $\left.\phi^{d}\right)$ the beam (resp. diffuse) component of the solar flux $\left(\mathrm{J} / \mathrm{m}^{2} . \mathrm{s}\right)$.

Heat transfers within opaque walls and glazings are described by partial differential equations (PDE) with scalar equivalent thermal parameters, $[9,36]$. Let $p \in \llbracket 1, N_{s} \rrbracket$ be the surface index, with $N_{s}$ the number of surfaces, and $\theta_{s}$ the corresponding temperature field. The temperature field $\theta_{s}$ is governed by the following equation:

$$
\left\{\begin{array}{l}
S_{s} \rho c_{s} \frac{\partial \theta_{s}}{\partial t}-S_{s} \frac{\partial}{\partial x}\left(k_{s} \frac{\partial \theta_{s}}{\partial x}\right)=0, \quad(x, t) \in\left[0, L_{s}\right] \times\left(0, t_{a}\right] \\
-k_{s} \frac{\partial \theta_{s}}{\partial x}(0, t)=\sum_{p=1}^{N_{s}}\left(\mathcal{R}_{s p}^{00}+\mathcal{R}_{s p}^{0 L}\right)+\mathcal{R}_{s}^{0 \infty}-\sum_{z=1}^{N_{z}} \mathcal{C}_{s z}^{0}+\overline{\mathcal{C}}_{s}^{0}+\underline{\mathcal{C}}_{s}^{0}+\Phi_{s}^{b 0}+\Phi_{s}^{d 0} \\
k_{s} \frac{\partial \theta_{s}}{\partial x}\left(L_{s}, t\right)=\sum_{p=1}^{N_{s}}\left(\mathcal{R}_{s p}^{L 0}+\mathcal{R}_{s p}^{L L}\right)+\mathcal{R}_{s}^{L \infty}-\sum_{z=1}^{N_{z}} \mathcal{C}_{s z}^{L}+\overline{\mathcal{C}}_{s}^{L}+\underline{\mathcal{C}}_{s}^{L}+\Phi_{s}^{b L}+\Phi_{s}^{d L} \\
\theta_{s}(x, t=0)=\theta_{s}^{0}(x)
\end{array}\right.
$$

All terms are described into table 2, they account for solar gains, convective gains through the environment, and radiative gains from adjacent facing surfaces and from the sky. We note $\alpha_{s p}^{0 L}$ the radiative exchange coefficient between the

\begin{tabular}{l|l} 
Flux & Definition \\
\hline $\mathcal{R}_{s p}^{00}=\alpha_{s p}^{00}\left(\theta_{p}(0, t)-\theta_{s}(0, t)\right)$ & \\
$\mathcal{R}_{s p}^{0 L}=\alpha_{s p}^{0 L}\left(\theta_{p}\left(L_{p}, t\right)-\theta_{s}(0, t)\right)$ & longwave radiation exchange between \\
$\mathcal{R}_{s p}^{L 0}=\alpha_{s p}^{L 0}\left(\theta_{p}(0, t)-\theta_{s}\left(L_{s}, t\right)\right)$ & adjacent surfaces facing each other \\
$\mathcal{R}_{s p}^{L L}=\alpha_{s p}^{L L}\left(\theta_{p}\left(L_{p}, t\right)-\theta_{s}\left(L_{s}, t\right)\right)$ & \\
\hline $\mathcal{R}_{s}^{0 \infty}=\beta_{s}^{0}\left(T^{\infty}-\theta_{s}(0, t)\right)$ & longwave radiation between surfaces and the \\
$\mathcal{R}_{s}^{L \infty}=\beta_{s}^{L}\left(T^{\infty}-\theta_{s}\left(L_{s}, t\right)\right)$ & sky \\
\hline$\overline{\mathcal{C}}_{s}^{0}=\bar{h}_{s}^{0}\left(T_{a}-\theta_{s}(0, t)\right)$ & convection exchange between surfaces and \\
$\overline{\mathcal{C}}_{s}^{L}=\bar{h}_{s}^{L}\left(T_{a}-\theta_{s}\left(L_{s}, t\right)\right)$ & outside environment \\
\hline$\overline{\mathcal{C}}_{s}^{0}=\underline{h}_{s}^{0}\left(T_{g}-\theta_{s}(0, t)\right)$ & conduction between surfaces and the ground \\
$\mathcal{\mathcal { C }}_{s}^{L}=\underline{h}_{s}^{L}\left(T_{g}-\theta_{s}\left(L_{s}, t\right)\right)$ & \\
\hline$\Phi_{s}^{b 0}=\alpha_{s}^{0} \gamma_{s}^{0 b} \phi^{b}$ & \\
$\Phi_{s}^{d 0}=\alpha_{s}^{0} \gamma_{s}^{0 d} \phi^{d}$ & \\
$\Phi_{s}^{b L}=\alpha_{s}^{L} \gamma_{s}^{L b} \phi^{b}$ & \\
$\Phi_{s}^{d L}=\alpha_{s}^{L} \gamma_{s}^{L d} \phi^{d}$ &
\end{tabular}

Table 2: Flux definition on the walls' boundaries.

face $(x=0)$ of surface $s$ and the face $\left(x=L_{p}\right)$ of surface $p\left(\mathrm{~J} / \mathrm{K} \cdot \mathrm{m}^{2} . \mathrm{s}\right), \beta_{s}^{0}$ (resp. $\left.\beta_{s}^{L}\right)$ the radiative exchange coefficient between the face $(x=0)\left(\operatorname{resp} .\left(x=L_{s}\right)\right)$ 
of surface $s$ and the sky $\left(\mathrm{J} / \mathrm{K} \cdot \mathrm{m}^{2} . \mathrm{s}\right), T^{\infty}$ the equivalent sky temperature $(\mathrm{K})$, $\bar{h}_{s}^{0}\left(\right.$ resp. $\left.\bar{h}_{s}^{L}\right)$ the convective heat exchange coefficient between the face $(x=0)$ (resp. $\left.\left(x=L_{s}\right)\right)$ of surface $s$ and the outside air $\left(\mathrm{J} / \mathrm{K} \cdot \mathrm{m}^{2} . \mathrm{s}\right), T_{a}$ the mean outside air temperature $(\mathrm{K}), \underline{h}_{s}^{0}\left(\operatorname{resp} . \underline{h}_{s}^{L}\right)$ the diffusive heat coefficient between the face $(x=0)$ (resp. $\left.\left(x=L_{s}\right)\right)$ of surface $s$ and the ground $\left(\mathrm{J} / \mathrm{K} \cdot \mathrm{m}^{2} . \mathrm{s}\right), T_{g}$ the ground temperature $(\mathrm{K}), \alpha_{s}^{0}$ (resp. $\left.\alpha_{s}^{L}\right)$ the absorbance of the face $(x=0)$ (resp. $\left.\left(x=L_{s}\right)\right)$ of surface $s, \gamma_{s}^{0 b}\left(\right.$ resp. $\left.\gamma_{s}^{0 d}\right)$ the exposure coefficient to the beam (resp. diffuse) solar component of the face $(x=0)$ of surface $s$, and $\phi^{b}$ (resp. $\left.\phi^{d}\right)$ the beam (resp. diffuse) component of the solar flux $\left(\mathrm{J} / \mathrm{m}^{2} . \mathrm{s}\right)$.

We also consider a model for the thermal behavior of heat devices inside the zones. Let $\Theta_{z}$ be the temperature of the heating device $z \in \llbracket 1, N_{z} \rrbracket$, given as the solution of the following ODE:

$$
\left\{\begin{array}{l}
d_{z} \frac{d \Theta_{z}}{d t}=\mathcal{P}_{z}-\mathcal{W}_{z} \\
\Theta_{z}(t=0)=\Theta_{z}^{0}
\end{array}\right.
$$

where $\mathcal{P}_{z}=\eta_{z} P_{z}$ is the supplied power, with $\eta_{z}$ the heating device efficiency and $P_{z}$ the input power $(\mathrm{J} / \mathrm{s})$.

PDEs are spatially discretized by the finite elements method with $P^{1}$ basis functions, and the time integration of the whole system is done by an implicit Euler scheme with a constant timestep $\Delta t=10 \mathrm{~min},[31,11]$.

\subsection{Case study}

The forecoming presentation of the model calibration method will be applied to a generic numerical test case. Actually, it represents an idealized two-zone building, see figure 1, with geometry and dimensions very similar to those defined in the BESTest testing protocol for dynamical thermal simulation softwares, [17]. Therefore, the test case may be viewed as a benchmark test for energy performance contracts.
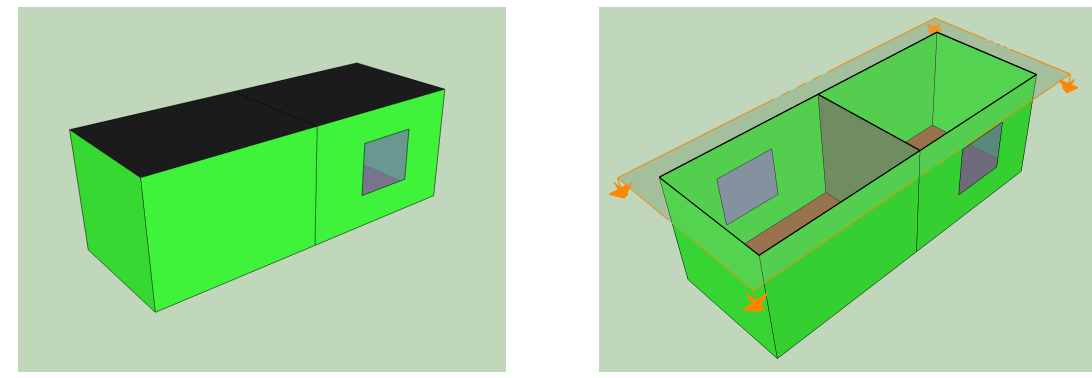

Figure 1: Geometric visualization of the case study on Google SketchUp CAD software.

The numerical tests will be done using weather data coming from EnergyPlus weather files corresponding to the city of Lyon (France), and we choose simple idealized occupancy scenarios. We get the sensor response from synthetic thermal data obtained by simulations based on model (1)-(2)-(3). 


\subsection{Quantities of interest}

As motivated in the introduction, the present energy performance assessment method strongly focuses on the possibility to accurately compute the building energy flux balance and energy consumption. These quantities are hereafter called quantities of interest and we detail their computations in the following sections.

\subsubsection{Intrinsic minimal consumption}

The first quantity of interest is the intrinsic minimal consumption, representing the building energy needs. We compute it as the power needed to reach a given setpoint temperature in the rooms. We thus solve an optimization problem where the supplied powers $P_{z}$ are the unknowns:

$$
\left\{P_{z}^{\star}\right\}_{z}=\underset{P_{z} \in L^{2}\left(0, t_{a}\right)}{\arg \min }\left\{\frac{1}{2} \sum_{z}\left\|T_{z}\left(P_{z}\right)-T^{c}\right\|_{\mathcal{M}_{c}}^{2}+\frac{\omega}{2} \sum_{z}\left\|P_{z}\right\|_{L^{2}\left(0, t_{a}\right)}^{2}\right\}
$$

This linear optimization problem is solved by the same tools as those used for the inverse method, which will be described in the next section. The parameter $\omega \in \mathbb{R}_{+}^{*}$ adjusts the severity of the minimal-norm constraints. Since the range of $P_{z}$ is about $10^{3}$, we chose $\omega=10^{-7}$ in our computations.

\subsubsection{Energy flux decomposition}

The second goal of the methodology is to derive a model that accurately computes the building energy flux balance. In order to evaluate this objective, we define the energy flux decomposition as a time-integration of the heat fluxes appearing in the zone state equation:

$$
C_{z} \frac{d T_{z}}{d t}=\sum_{r=1}^{N_{z}} \mathcal{A}_{r z}+\overline{\mathcal{A}}_{z}+\sum_{s=1}^{N_{s}} S_{s}\left(\mathcal{C}_{s z}^{0}+\mathcal{C}_{s z}^{L}\right)+\mathcal{Q}_{z}+\mathcal{W}_{z}+\Phi_{z}^{b}+\Phi_{z}^{d}
$$

with the terms described in table 3 . Since the internal gains $\mathcal{Q}_{z}$ and the interzone thermal coupling $\mathcal{A}_{r z}$ are supposed to be known, they will not be part of this decomposition. Only the last flux $\mathcal{N}$ does not appear in equation (5), and represent the energy loss related to heating device efficiency.

\begin{tabular}{l|l} 
Flux & Description \\
\hline$\overline{\mathcal{A}}=\sum_{z} c_{a} q_{z}\left(T_{a}-T_{z}\right)$ & air renewal \\
\hline $\begin{array}{c}\mathcal{C}^{s}=\sum_{s, z} S_{s} h_{s z}^{0}\left(\theta_{s}(0, t)-T_{z}\right) \\
\quad+\sum_{s, z} S_{s} h_{s z}^{L}\left(\theta_{s}\left(L_{s}, t\right)-T_{z}\right)\end{array}$ & convective coupling with the walls \\
\hline $\mathcal{W}=\sum_{z} \lambda_{z}\left(\Theta_{z}-T_{z}\right)$ & convective coupling with heaters \\
\hline$\Phi=\sum_{z} A_{z} \tau_{z}\left(\gamma_{z}^{b} \phi^{b}+\gamma_{z}^{d} \phi^{d}\right)$ & solar gains \\
\hline $\mathcal{N}=\sum_{z}\left(\eta_{z}-1\right) P_{z}$ & loss related to heating device efficiency
\end{tabular}

Table 3: Description of heat flux in the zone. 
This quantity allows to distinguish the components of all heat gains and losses of the building. It often takes the form of a Sankey diagram, and is the cornerstone of the renovation proposals and the energy performance contracts.

\section{Parameter identification method}

For detailed models and cases involving time or space dependent functions within the unknowns, model identification can be cumbersome. Indeed, the computation of the cost-function's gradient requires to compute several times the overall building thermal state. The optimal control theory gives a general mathematical framework to apprehend this type of problems, [21]. In particular, the introduction of an adjoint model leads to a fast computation of the costfunction's gradient.

\subsection{Measurements}

We describe here the measurements available for the energy performance assessment method. We require the measure of weather data, use conditions, the power supplied to each heating systems, the zone mean air temperatures, the heating systems surface temperatures, and some wall surface temperatures. Actually, we only require the wall surface temperature measurements for one wall per type of walls (heavy walls, light walls, ...).

We get data from an on-site sensors deployment, see figure 2. They are composed of 2 zone air temperature sensors, 1 per room, and 6 surface temperature sensors, 1 per heating device, 2 for both surfaces of a heavy wall and 2 for both surfaces of the light wall.

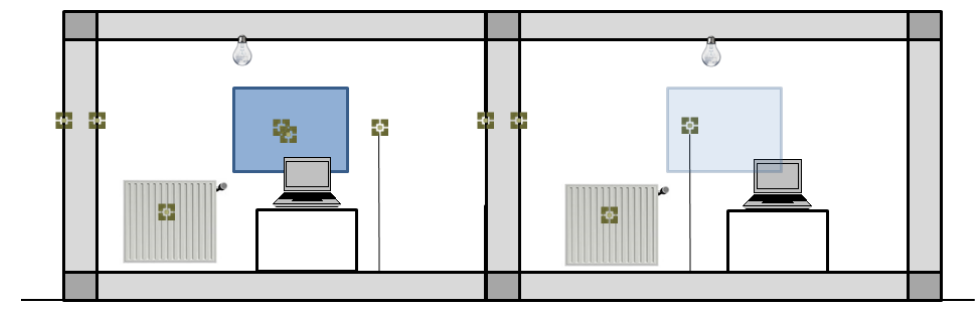

Figure 2: Thermal sensors in the two-zone test case.

\subsection{Choice of the unknown parameters}

In a nonlinear model identification procedure, one can't determine all parameters of a complex model without accurate a priori values, especially with building thermal modeling in which we deal with a big number of parameters. The first step of this process is then to select the unknown parameters of the inverse problem as a compromise between the quantity and the quality of measurements, and the type and the number of unknowns. 
Here we use the results of a sensitivity analysis of building thermal parameters [14] coupled with the conclusions of the studies [25, 19] on the parameters identifiability. We also want to accurately reconstruct the main energy flux of the building thus the unknown parameters have to be related thereof. We can thus argue that, among all parameters, those which are both uncertain, thermally influent and identifiable at the same time are the heating system properties $\left(d_{z}, \eta_{z}, \lambda_{z}\right)$, the wall internal thermal parameters $\left(\rho c_{s}, k_{s}\right)$, the room air renewals $q_{z}$ and the window transmittances $\tau_{z}$. However, the simultaneous identification of time-dependent room air renewals $q_{z}$ and window transmittances $\tau_{z}$ is not possible from the selected data and observation points, so we decide to identify mean air renewals $\bar{q}_{z}$ :

$$
\bar{q}_{z}=\frac{1}{t_{a}} \int_{0}^{t_{a}} q_{z}(t) d t
$$

We obtain the following vector of unknowns:

$$
u=\left\{\rho c_{s}, k_{s}, \eta_{z}, d_{z}, \lambda_{z}, \bar{q}_{z}, \tau_{z}\right\}_{s, z}
$$

\subsection{Inverse problem}

We describe here the inverse method used in this work. Let $\mathcal{U}$ be the space of unknown parameters and $\mathcal{S}$ the space of thermal states verifying the thermal model (1)-(2)-(3). We introduce the operator $f$ which evaluates the thermal state at any space-time point:

$$
f:\left(\begin{array}{rl}
\mathcal{U} & \longrightarrow \mathcal{S} \\
u & \longmapsto
\end{array}(u)=\left\{\theta_{s}(x, t), T_{z}(t), \Theta_{z}(t)\right\}_{s, z}(u), \quad \forall(x, t)\right.
$$

Let $\mathcal{M}$ be the observations space. We define the observation operator $O \in$ $\mathscr{L}(\mathcal{S}, \mathcal{M})$, and the composed operator $\hat{f}$ by:

$$
\hat{f}: \mathcal{U} \stackrel{f}{\longrightarrow} \mathcal{S} \stackrel{O}{\longrightarrow} \mathcal{M}
$$

It means that $\hat{f}$ evaluates the thermal response of the model at the observation points.

Using optimal control theory, the parameter identification problem can be formulated as a minimization problem, where the unknowns are sought as minimizers of a cost-function evaluating the gap between measurements and computed physical state, [20]. Such inverse problems are generally ill-posed as defined by Hadamard, which means that their solution, if it exists and is unique, does not continuously depend on data, [35]. In order to obtain a stable numerical scheme, some regularization has to be introduced. We use here Tikhonov regularization, [13], which consists in penalizing the cost-function by a quadratic regularization term, thus providing local convexity and transforming the initial problem into a well-posed one, [10]. The cost-function involved in the optimization problem writes:

$$
J(u)=\frac{1}{2}\|\hat{f}(u)-m\|_{\mathcal{M}}^{2}+\frac{\epsilon}{2}\left\|u-u^{0}\right\|_{\mathcal{U}}^{2}+\frac{\mu}{2}\|G u-c\|_{\mathcal{C}}^{2}
$$


The first term, called fidelity term, evaluates the discrepancy between data $m \in \mathcal{M}$ and the model response at observation points. The second one is the so-called Tikhonov regularization term, with $u^{0}$ an a priori estimate of the unknowns and $\epsilon$ the regularization parameter. The ill-posed nature of inverse problems involving the identification of intrinsic thermal parameters is soft compared to heat source estimations problems. We thus chose an heuristic small value $\epsilon=1 e^{-6}$ in our computations. The last term enforces the minimization algorithm to take constraints into account. We note $\mathcal{C}$ the constraints space, $c \in \mathcal{C}$ a constraint and $G \in \mathscr{L}(\mathcal{U}, \mathcal{C})$ the constraints operator. This so-called penalization term allows to take into account some relations between parameters.

Finally, the inverse problem reads:

$$
\text { find } u \in \mathcal{U} \text { such that } u=\underset{v \in \mathcal{U}}{\arg \min } J(v)
$$

The direct thermal state (1)-(2)-(3) is nonlinear with respect to some components of $u$. Thus we need to use a nonlinear optimization method to solve (11), and we chose the widely-used Levenberg-Marquardt method. This algorithm relies on an iterative linearizations process which solves, at each iteration, a quadratic subproblem based on the first order Taylor expansion $\hat{f}^{\prime}(u)=\left\{\delta \theta_{s}, \delta T_{z}, \delta \Theta_{z}\right\}_{s, z}$ of the nonlinear thermal state $f(u)$, [24]. The subproblem minimizations are done using the conjugate gradient method, and the cost-function gradient is computed with the adjoint state method, [21, 11, 7]. Coming from the control theory, the adjoint state method gives an explicit expression for the adjoint operator $\hat{f}^{\prime}(u)^{*}=\left\{\delta \theta_{s}^{*}, \delta T_{z}^{*}, \delta \Theta_{z}^{*}\right\}_{s, z}$ of the first order Taylor expansion $\hat{f}^{\prime}(u) \in \mathscr{L}(\mathcal{U}, \mathcal{M})$. It is the solution of equation (13), with $\varpi_{s}^{x}$ the function defined for $x \in\left\{0, L_{s}\right\}$ by:

$$
\varpi_{s}^{x}= \begin{cases}\theta_{s}(x, t)+\delta \theta_{s}(x, t)-\theta_{s}^{d}(x, t), & \text { if surface } s \text { has sensors } \\ 0, & \text { else }\end{cases}
$$

See [8] for more details about these algorithm and numerical methods applied to the nodal multizone model. The different terms appearing in the adjoint model (13) are defined in table 4. 


$$
\left\{\begin{array}{l}
\left\{\begin{array}{l}
-C_{z} \frac{d \delta T_{z}^{*}}{d t}=\sum_{r=1}^{N_{z}} \delta \mathcal{A}_{r z}^{*}-c_{a} q_{z} \delta T_{z}^{*}+\sum_{s=1}^{N_{s}} S_{s}\left(\delta \mathcal{C}_{s z}^{0 *}+\delta \mathcal{C}_{s z}^{L *}\right)+\delta \mathcal{W}_{z}^{*}+\left(T_{z}+\delta T_{z}-T_{z}^{d}\right) \\
\delta T_{z}^{*}\left(t=t_{a}\right)=0
\end{array}\right. \\
\left\{\begin{array}{l}
-d_{z} \frac{d \delta \Theta_{z}^{*}}{d t}=-\delta \mathcal{W}_{z}^{*}+\left(\Theta_{z}+\delta \Theta_{z}-\Theta_{z}^{d}\right) \\
\delta \Theta_{z}^{*}\left(t=t_{a}\right)=0
\end{array}\right. \\
\left\{\begin{array}{l}
-S_{s} \rho c_{s} \frac{\partial \delta \theta_{s}^{*}}{\partial t}-S_{s} \frac{\partial}{\partial x}\left(k_{s} \frac{\partial \delta \theta_{s}^{*}}{\partial x}\right)=0, \quad(x, t) \in\left[0, L_{s}\right] \times\left(0, t_{a}\right] \\
-k_{s} \frac{\partial \delta \theta_{s}^{*}}{\partial x}(0, t)=\sum_{p=1}^{N_{s}}\left(\delta \mathcal{R}_{s p}^{00 *}+\delta \mathcal{R}_{s p}^{0 L *}\right)-\sum_{z=1}^{N_{z}} \delta \mathcal{C}_{s z}^{0 *}-\left(\beta_{s}^{0}+\bar{h}_{s}^{0}+\underline{h}_{s}^{0}\right) \delta \theta_{s}^{*}(0, t)+\varpi_{s}^{0} \\
k_{s} \frac{\partial \delta \theta_{s}^{*}}{\partial x}\left(L_{s}, t\right)=\sum_{p=1}^{N_{s}}\left(\delta \mathcal{R}_{s p}^{L 0 *}+\delta \mathcal{R}_{s p}^{L L *}\right)-\sum_{z=1}^{N_{z}} \delta \mathcal{C}_{s z}^{L *}-\left(\beta_{s}^{L}+\bar{h}_{s}^{L}+\underline{h}_{s}^{L}\right) \delta \theta_{s}^{*}\left(L_{s}, t\right)+\varpi_{s}^{L_{s}} \\
\delta \theta_{s}^{*}\left(x, t=t_{a}\right) \equiv 0
\end{array}\right.
\end{array}\right.
$$

\begin{tabular}{l|l} 
Flux & Definition \\
\hline$\delta \mathcal{A}_{r z}^{*}=c_{a} R_{z r}\left(\delta T_{r}^{*}-\delta T_{z}^{*}\right)$ & interzone airmass exchange \\
\hline$\delta \mathcal{C}_{s z}^{0 *}=h_{s z}^{0}\left(\delta \theta_{s}^{*}(0, t)-\delta T_{z}^{*}\right)$ & convection between surfaces and inside air \\
$\delta \mathcal{C}_{s z}^{L *}=h_{s z}^{L}\left(\delta \theta_{s}^{*}\left(L_{s}, t\right)-\delta T_{z}^{*}\right)$ & \\
\hline$\delta \mathcal{R}_{s p}^{00 *}=\alpha_{s p}^{00}\left(\delta \theta_{p}^{*}(0, t)-\delta \theta_{s}^{*}(0, t)\right)$ & \\
$\delta \mathcal{R}_{s p}^{0 L *}=\alpha_{s p}^{0 L}\left(\delta \theta_{p}^{*}\left(L_{p}, t\right)-\delta \theta_{s}^{*}(0, t)\right)$ & long wave radiation between adjacent \\
$\delta \mathcal{R}_{s p}^{L 0 *}=\alpha_{s p}^{L 0}\left(\delta \theta_{p}^{*}(0, t)-\delta \theta_{s}^{*}\left(L_{s}, t\right)\right)$ & surfaces \\
$\delta \mathcal{R}_{s p}^{L L *}=\alpha_{s p}^{L L}\left(\delta \theta_{p}^{*}\left(L_{p}, t\right)-\delta \theta_{s}^{*}\left(L_{s}, t\right)\right)$ & \\
\hline$\delta \mathcal{W}_{z}^{*}=\lambda_{z}\left(\delta \Theta_{z}^{*}-\delta T_{z}^{*}\right)$ & convective gains from heaters
\end{tabular}

Table 4: Flux definition for the adjoint model.

The gradient of the cost-function is then obtained by the following explicit 
expressions:

$$
\begin{aligned}
\left.\nabla \tilde{J}_{u}(\delta u)\right|_{\rho c_{s}} & =-\int_{\mathcal{Q}_{s}} S_{s} \frac{\partial \theta_{s}}{\partial t} \delta \theta_{s}^{*}+\epsilon\left(\rho c_{s}+\delta \rho c_{s}-\rho c_{s}^{0}\right)+\mu \delta \rho c_{s}^{C}+\nu \delta \rho c_{s} \\
\left.\nabla \tilde{J}_{u}(\delta u)\right|_{k_{s}} & =-\int_{\mathcal{Q}_{s}} S_{s} \frac{\partial \theta_{s}}{\partial x} \frac{\partial \delta \theta_{s}^{*}}{\partial x}+\epsilon\left(k_{s}+\delta k_{s}-k_{s}^{0}\right)+\mu \delta k_{s}^{C}+\nu \delta k_{s} \\
\left.\nabla \tilde{J}_{u}(\delta u)\right|_{\eta_{z}} & =\int_{0}^{t_{a}} P_{z} \delta \Theta_{z}^{*}+\epsilon\left(\eta_{z}+\delta \eta_{z}-\eta_{z}^{0}\right)+\nu \delta \eta_{z} \\
\left.\nabla \tilde{J}_{u}(\delta u)\right|_{d_{z}} & =-\int_{0}^{t_{a}} \frac{d \Theta_{z}}{d t} \delta \Theta_{z}^{*}+\epsilon\left(d_{z}+\delta d_{z}-d_{z}^{0}\right)+\nu \delta d_{z} \\
\left.\nabla \tilde{J}_{u}(\delta u)\right|_{\lambda_{z}} & =\int_{0}^{t_{a}}\left(\Theta_{z}-T_{z}\right)\left(\delta T_{z}^{*}-\delta \Theta_{z}^{*}\right)+\epsilon\left(\lambda_{z}+\delta \lambda_{z}-\lambda_{z}^{0}\right)+\nu \delta \lambda_{z} \\
\left.\nabla \tilde{J}_{u}(\delta u)\right|_{\bar{q}_{z}} & =\int_{0}^{t_{a}} c_{a}\left(T_{a}-T_{z}\right) \delta T_{z}^{*}+\epsilon\left(\bar{q}_{z}+\delta \bar{q}_{z}-\bar{q}_{z}^{0}\right)+\nu \delta \bar{q}_{z} \\
\left.\nabla \tilde{J}_{u}(\delta u)\right|_{\tau_{z}} & =\int_{0}^{t_{a}} A_{z}\left(\gamma_{z}^{b} \phi_{z}^{b}+\gamma_{z}^{d} \phi_{z}^{d}\right) \delta T_{z}^{*}+\epsilon\left(\tau_{z}+\delta \tau_{z}-\tau_{z}^{0}\right)+\nu \delta \tau_{z}
\end{aligned}
$$

with $\mathcal{Q}_{s}=\left[0, t_{a}\right] \times\left[0, L_{s}\right]$. The superscript $C$ represents the constraints term gradient computation $G^{T}(G u+G \delta u-c)$, see [8].

\subsection{Results}

We present in this section simulation results showing evidence of the performance of this approach. The results are obtained numerically with the following protocol: first, the state observations are generated with our own thermal model, but with a time-dependent air renewal $q_{z}(t)$; second, we disrupt the target parameters using uncertainty information coming from standard manual data collection; and third, we run the identification process using the generated state observations.

We first recall the classical uncertainties for the unknown parameters of the building thermal model in table $5,[22,14,33]$. The uncertainty value, in percentage, corresponds to a standard deviation. Since we're using a gradient descent to solve the optimization problem, our method may depend on the initialization of the algorithm. That's why we generate a Monte-Carlo sample of 50 randomly chosen parameters from table 5 . This sample constitutes 50 uncertain standard manual data collections, and we use them as initializations for our parameter identification method. The results are analyzed with the statistical tools described in table 6 .

The numerical experiment takes place in the city of Lyon (France) during the first week of February 2014, and we suppose that we have 6 days of measurements. We present in figures 3, 4 and 5 the weather conditions coming from EnergyPlus weather files and the use scenarios.

Results are presented in table 7. The identification process ran well for every initializations since mean errors $\bar{E}_{\%}$ of reconstructed values are lower than classical ones. The wall thermophysical properties are very accurately identified, 


\begin{tabular}{c|c|l} 
Parameter & Uncertainty $(\%)$ & Explanation \\
\hline$\rho c_{z}$ & 25 & internal composition of walls (built, insulation) \\
$k_{s}$ & 25 & internal composition of walls (built, insulation) \\
$\eta_{z}$ & 10 & type and characteristics of heating devices \\
$d_{z}$ & 40 & type and characteristics of heating devices \\
$\lambda_{z}$ & 30 & type, features and heaters location \\
$q_{z}$ & 50 & weather and walls' permeability \\
$\tau_{z}$ & 15 & glazing type
\end{tabular}

Table 5: Classical uncertainties in building thermal modeling.

\begin{tabular}{c|l|c} 
Parameter & Definition & Computation \\
\hline$u_{t a r}$ and $u_{r e c}$ & target and reconstructed values & \\
$\bar{u}_{r e c}$ & mean reconstructed value & \\
$\bar{E}$ & mean relative error $\bar{u}_{r e c}$ & $\frac{\left|\bar{u}_{r e c}-u_{t a r}\right|}{u_{t a r}}$ \\
$\bar{E}_{\%}$ & mean relative error in percentage & $\bar{E} \times 100$ \\
$\sigma_{\%}$ & standard deviation in percentage & $\sigma\left(\frac{u_{r e c}}{\bar{u}_{r e c}}\right) \times 100$
\end{tabular}

Table 6: Statistical tools.
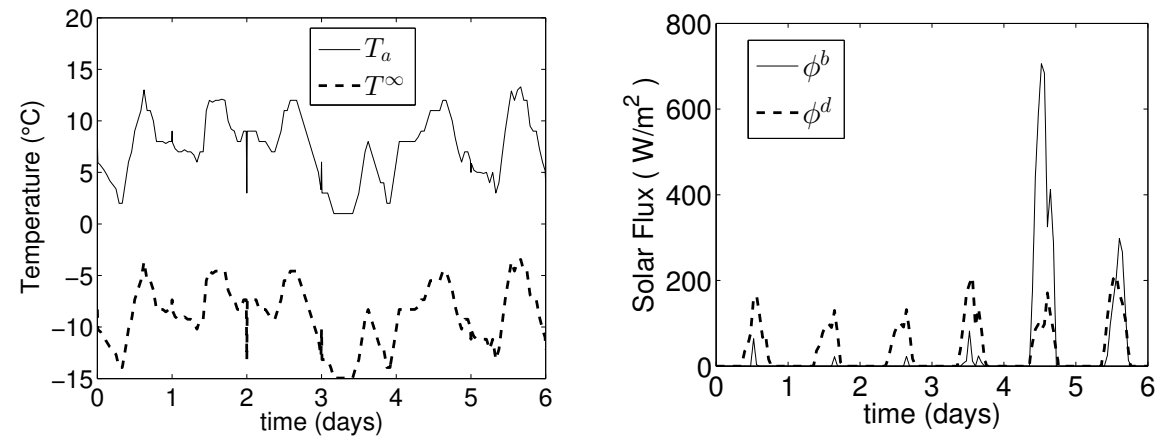

Figure 3: Weather data for the first week of February.

with standard deviations less than $0.7 \%$. The heating device efficiencies $\eta_{z}$ and convective coupling coefficients $\lambda_{z}$ are also improved, with an uncertainty going down to $2.4 \%$. The heating device capacities $d_{z}$ is improved by a factor 2 . The average rates of air renewal $\bar{q}_{z}$ and the window transmittances $\tau_{z}$ are not improved. This may be explain by the lack of thermal sensitivity of the chosen thermal model (1)-(2)-(3) with respect these parameters.

For each parameter set from the Monte-Carlo sample, we compute the thermal response of the model for the first zone. We obtain a collection of thermal responses, and we plot its extreme bounds in figure 6 . We can see that the ther- 

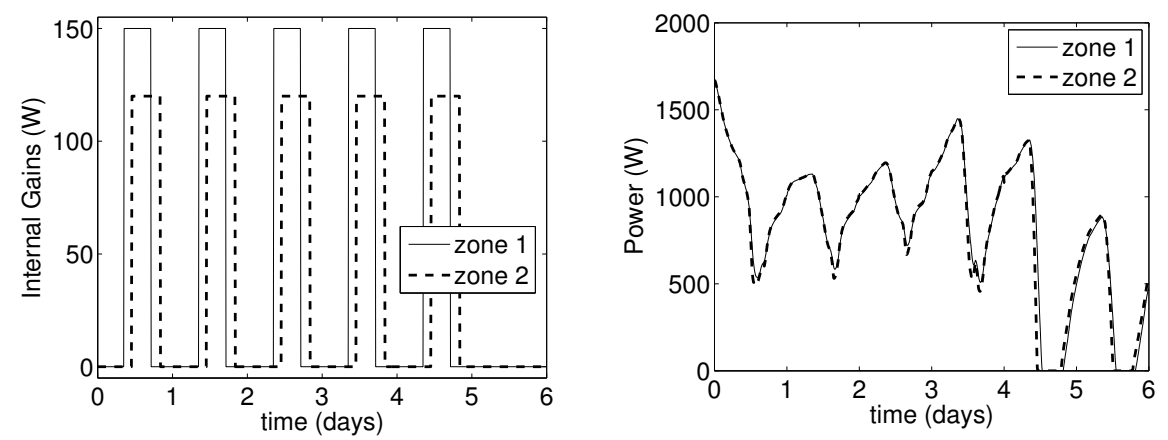

Figure 4: Use data $Q_{z}$ and heating systems' supplied power $P_{z}$ for the first week of February.

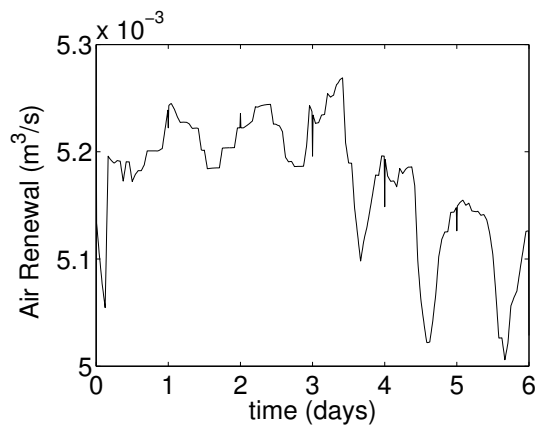

Figure 5: Time-dependent air renewal $q_{z}$ for the first week of February.

\begin{tabular}{c|c||c|c|l||c|c|l}
\multirow{2}{*}{ Parameter } & \multicolumn{2}{|c||}{ Target } & \multicolumn{3}{c||}{ Standard method } & \multicolumn{3}{c}{ Developed method } \\
& $u_{\text {tar }}$ & $\bar{u}$ & $\bar{E}_{\%}$ & $\sigma_{\%}$ & $\bar{u}_{\text {rec }}$ & $\bar{E}_{\%}$ & $\sigma_{\%}$ \\
\hline$\rho c_{s}$ & $1.800 \mathrm{e}^{6}$ & $1.713 \mathrm{e}^{6}$ & 4.8 & 21.2 & $1.797 \mathrm{e}^{6}$ & 0.1 & 0.2 \\
$k_{s}$ & 0.96 & 0.91 & 5.2 & 24.3 & 0.96 & 0 & 0.7 \\
$\eta_{z}$ & 0.900 & 0.897 & 0.3 & 6 & 0.900 & 0 & 2.4 \\
$d_{z}$ & 1000 & 1005.4 & 0.5 & 41.2 & 1005.8 & 0.6 & 18.9 \\
$\lambda_{z}$ & 20 & 19.56 & 2.2 & 29.4 & 20.00 & 0 & 2.4 \\
$\bar{q}_{z}$ & $5.1 \mathrm{e}^{-3}$ & $5.1 \mathrm{e}^{-3}$ & 0.9 & 30.4 & $5.2 \mathrm{e}^{-3}$ & 2 & 32.9 \\
$\tau_{z}$ & 0.500 & 0.494 & 1.2 & 11.3 & 0.500 & 0 & 12.1
\end{tabular}

Table 7: Target, mean and standard deviation of reconstructed values with the Monte-Carlo sample.

mal state uncertainty is reduced by a factor 10 , going from $\pm 2{ }^{\circ} \mathrm{C}$ to $\pm 0.2^{\circ} \mathrm{C}$. Since all unknown parameters in $u$ are intrinsic thermal properties, once we have identified them we can compute the thermal response over few days after 
measurements, see figure 6 . As expected, we observe that the improvement of the model response stays in time. Moreover, the low thermal error observed highlights the fact that the unknown parameters which kept their initial uncertainties (mainly the mean rates of air renewal, and the heating device capacities and convective coupling) have only a minor thermal influence.
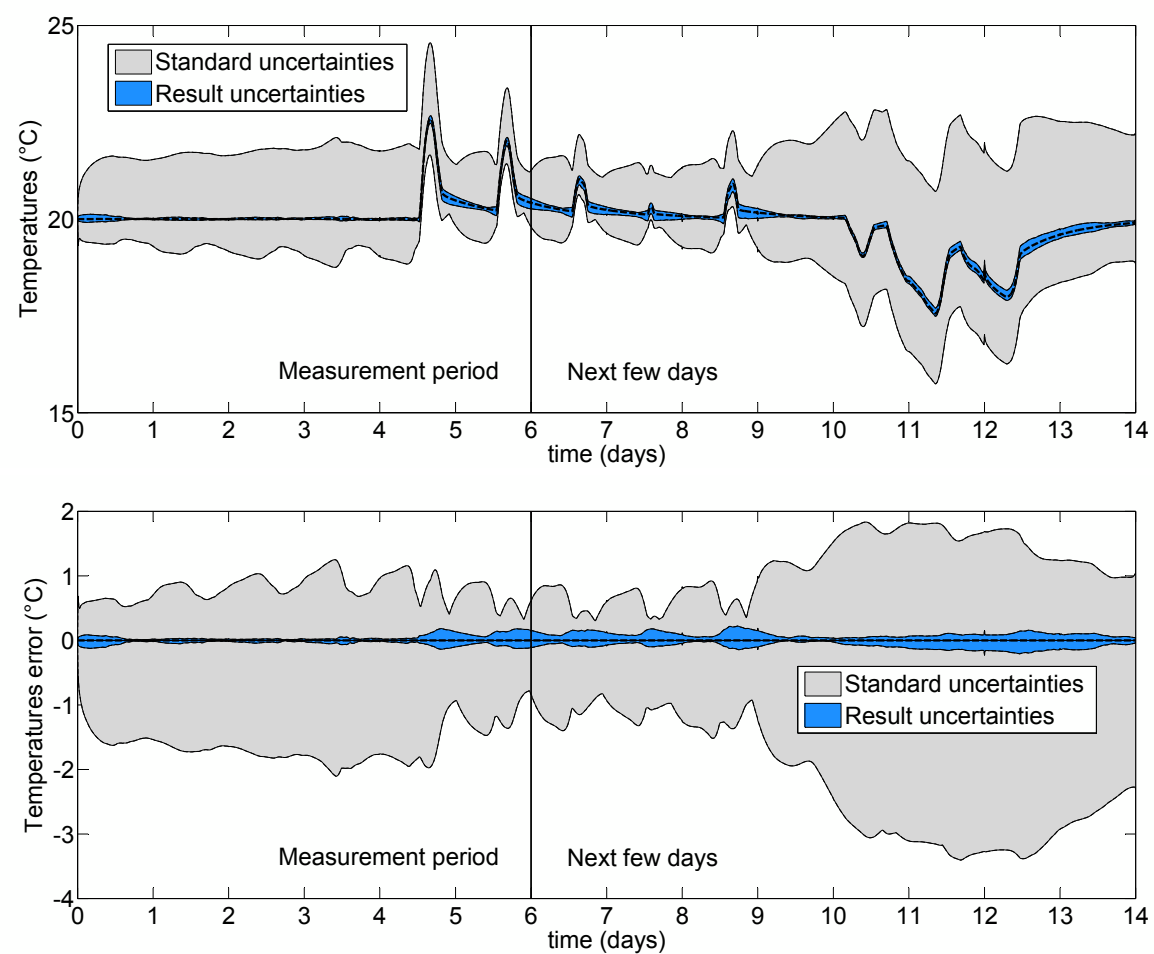

Figure 6: Extreme bounds of thermal response of the identified models for the first zone during the measurement days and some days after. On top, the temperatures ; on bottom, the error.

We can now compute the quantities of interest previously introduced to analyze the results. We show in table 8 the intrinsic minimal consumption corresponding to the standard approach and our inverse method. We can see in this table that we strongly reduce the initial uncertainty, going from a $9 \%$ standard deviation to less than $0.4 \%$. Thus the energy performance assessment method seems to reconcile the consumption evaluation.

As for the thermal response of the room, we can plot the extreme bounds of the total supplied power for all heating devices. This is done in figure 7 . Since this quantity of interest directly depends on the room temperatures, we recover the accuracy of the reconstructed thermal state in the consumption computation. Here, in the winter season, the standard method error on the 


\begin{tabular}{l|c|l|l} 
Minimal intrinsic consumption & Value $\left(\mathrm{kWh} / \mathrm{m}^{2}\right.$. year $)$ & $\bar{E}_{\%}$ & $\sigma_{\%}$ \\
\hline Target & 373.05 & - & - \\
Standard method & 367.94 & 1.4 & 9 \\
Developed method & 373.05 & 0 & 0.4
\end{tabular}

Table 8: Time-integration of the minimal intrinsic consumption reconstructions.

total power supplied is about $600 \mathrm{~W}$, which represents the heat gain of a small heating device. On the other side, our calibration method reduces the intrinsic minimal consumption uncertainty to less than $40 \mathrm{~W}$.
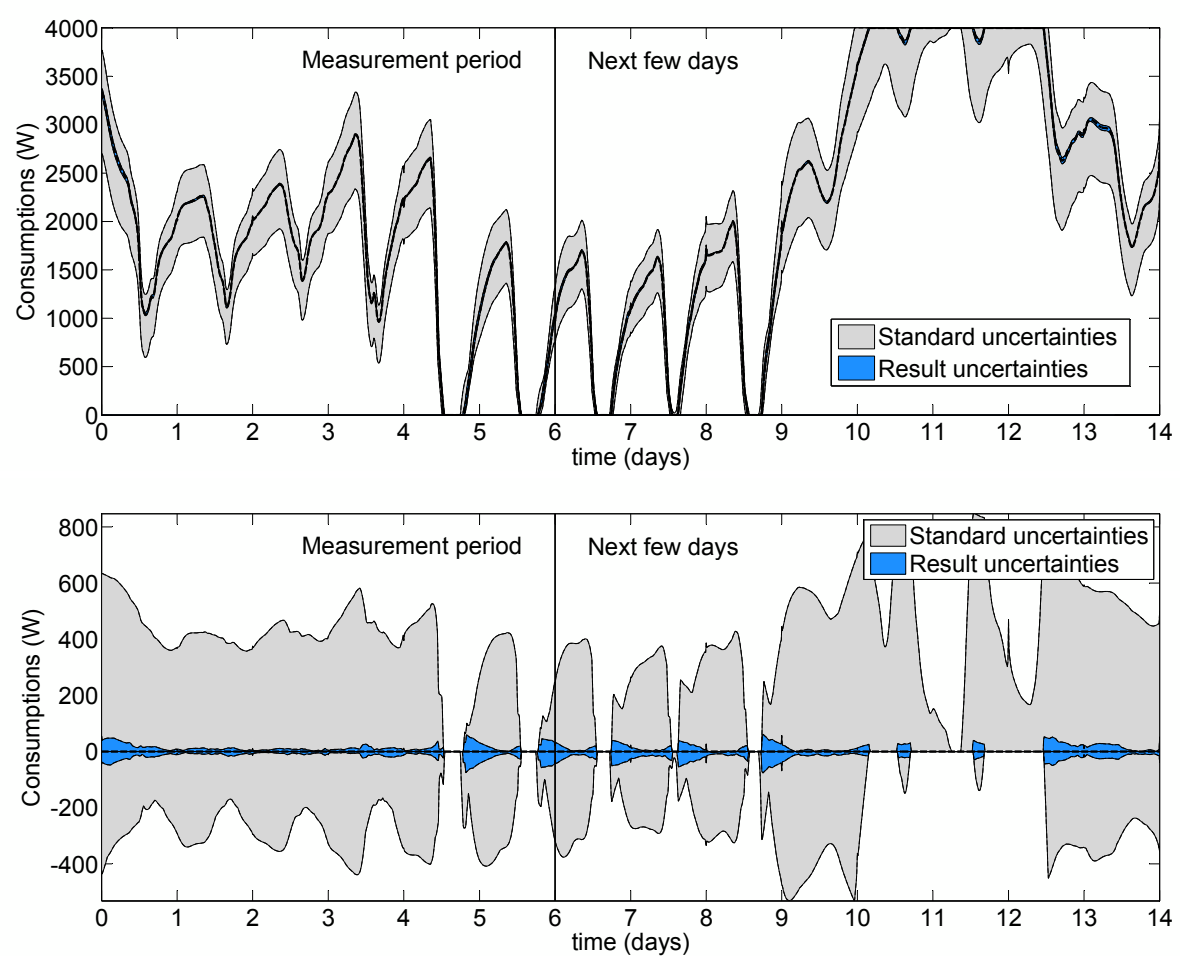

Figure 7: Extreme bounds of computed intrinsic minimal consumption of the identified models during the measurement days and the week after. On top, the consumption ; on bottom, the error.

The accuracy of the reconstructed temperature and energy consumption profiles are similar to other learning or calibration based diagnosis method. The advantage of our inverse thermal model is that it allows to calibrate the energy flux decomposition of the building, thus helping to get a detailed overview 
of the building energy performance where other approaches output global energy consumption value or index. This information is meaningful to helping of professionals in the choice of a renovation scenario.

We now compute the energy flux decomposition. The results are presented in table 9. Even if some unknown parameters kept an important uncertainty after the identification process, we can see that the energy balance uncertainties are reduced by our method. The high uncertainties with the standard method may lead to non-optimal refurbishment proposals, since the return on investment could be badly estimated. In our estimation method, the smaller uncertainties allow a more accurate computation of the return on investment, helping to find the optimal renovation scenarios. Moreover, it affords to propose trustful energy performance contracts.

\begin{tabular}{c|c|r|r|r|r} 
Method & Flux $\left(\mathrm{kWh} / \mathrm{m}^{2}\right.$.year $)$ & Target & Value & $\bar{E}_{\%}$ & $\sigma_{\%}$ \\
\hline \multirow{3}{*}{ Standard method } & $\int \overline{\mathcal{A}}$ & -54.37 & -53.83 & 1 & 29.6 \\
& $\int \mathcal{C}^{s}$ & -496.23 & -489.87 & 1.3 & 5.3 \\
& $\int \mathcal{N}$ & -37.30 & -38.58 & 3.4 & 56.2 \\
& $\int \Phi$ & 42.20 & 41.69 & 1.2 & 11.3 \\
Developed method & $\int \mathcal{W}$ & 335.73 & 329.34 & 1.9 & 8 \\
\hline & $\int \overline{\mathcal{A}}$ & -54.37 & -55.06 & 1.3 & 30.9 \\
& $\int \mathcal{C}$ & -496.23 & -495.76 & 0.1 & 1 \\
& $\int \mathcal{N}$ & -37.30 & -37.23 & 0.2 & 21.5 \\
& $\int \mathcal{W}$ & 42.20 & 42.33 & 0.3 & 6.9 \\
& $\int \mathcal{W}$ & 335.73 & 335.82 & 0 & 2.8
\end{tabular}

Table 9: Target, mean and standard deviation of the energy flux decomposition reconstructions.

\section{Applications and performance analysis}

In this section, we study the performance of the energy diagnosis method with respect to the initial uncertainties, the measurement noise and the season of the diagnosis. After that, we apply the method on sensor data coming from an EnergyPlus simulation of the building. This last step approaches the real case application on a building with thermal measurements. Here again, we assess the model capacity to accurately predict the quantities of interest.

\subsection{High initial uncertainties on the unknowns}

In this study, we analyze the parameter identification results when the initial uncertainties on the unknowns $u$ are high. It is motivated by the fact that the real uncertainties may be higher than the one used in the case of existing building. Moreover, we may reasonably estimate that the develop energy performance assessment method lays on the idea of easing the on-site data gathering process. We thus decide to put a $50 \%$ uncertainty on each unknown parameter. 
Then we reproduce the Monte-Carlo sample of 50 randomly chosen parameters with these new (larger) uncertainties, and run the parameter identification method.

The table 10 presents the identification results. We can see that the initial uncertainties are higher than the one previously used. The results for the wall internal heat parameters $\left(k_{s}\right.$ and $\left.\rho c_{s}\right)$ are very good, starting from a $50 \%$ uncertainty to $1.6 \%$ and $0.5 \%$ respectively. The heating device efficiencies $\eta_{z}$ and convective coupling coefficients $\lambda_{z}$ are also correctly reconstructed with an uncertainty less than $5.5 \%$. As previously remarked, the heating system capacities $d_{z}$, the mean air renewals $\bar{q}_{z}$ and the window transmittances $\tau_{z}$ are not sufficiently thermally influent to be as much improved.

\begin{tabular}{c|c||c|l|l||c|l|l}
\multirow{2}{*}{ Parameter } & \multicolumn{2}{|c||}{ Target } & \multicolumn{3}{c||}{ Highly uncertain case } & \multicolumn{3}{c}{ Developed method } \\
& $u_{\text {tar }}$ & $\bar{u}$ & $\bar{E}_{\%}$ & $\sigma_{\%}$ & $\bar{u}_{r e c}$ & $\bar{E}_{\%}$ & $\sigma_{\%}$ \\
\hline$\rho c_{s}$ & $1.800 \mathrm{e}^{6}$ & $1.909 \mathrm{e}^{6}$ & 6 & 52.6 & $1.803 \mathrm{e}^{6}$ & 0.2 & 0.5 \\
$k_{s}$ & 0.960 & 0.977 & 1.7 & 56.4 & 0.959 & 0.1 & 1.6 \\
$\eta_{z}$ & 0.900 & 0.850 & 5.9 & 49.6 & 0.918 & 2 & 5 \\
$d_{z}$ & 1000 & 1085.7 & 8.6 & 35.2 & 930.8 & 6.9 & 36.4 \\
$\lambda_{z}$ & 20 & 21.62 & 8.1 & 51.6 & 20.44 & 2.2 & 5.5 \\
$\bar{q}_{z}$ & $5.1 \mathrm{e}^{-3}$ & $5.6 \mathrm{e}^{-3}$ & 9.5 & 45.1 & $6.7 \mathrm{e}^{-3}$ & 32.2 & 53.4 \\
$\tau_{z}$ & 0.500 & 0.576 & 15.3 & 46.9 & 0.576 & 15.2 & 26.6
\end{tabular}

Table 10: Parameter identification results when considering high initial uncertainties on the unknowns $u$.

Here again, we plot in figure 8 extreme bounds of the thermal response of the first zone obtained with these results, and their error graph. It illustrates the robustness of the method with respect to the initialization, since the thermal state's uncertainty is reduced from $\pm 15^{\circ} \mathrm{C}$ to less than $\pm 1^{\circ} \mathrm{C}$.

After that, we compute the quantities of interest corresponding to these results. The minimal intrinsic consumption computations in the uncertain case and after the identification process are presented in table 11. In the uncertain case, the consumption are given with a high uncertainty of $50.6 \%$. Even if some parameters kept their high initial uncertainty, we can see that the calibration process introduced in our energy performance assessment method allows the reduction of this uncertainty to $1.4 \%$. So the calibrated computation of the intrinsic minimal consumption appears to work very well.

\begin{tabular}{l|c|l|l} 
Minimal intrinsic consumption & Value $\left(\mathrm{kWh} / \mathrm{m}^{2}\right.$. year $)$ & $\bar{E}_{\%}$ & $\sigma_{\%}$ \\
\hline Target & 373.05 & - & - \\
Highly uncertain case & 378.78 & 1.5 & 50.6 \\
Developed method & 374.61 & 0.4 & 1.4
\end{tabular}

Table 11: Time-integration of the minimal intrinsic consumption reconstruction.

We plot in figure 9 the extreme bounds of the reconstructed intrinsic minimal 

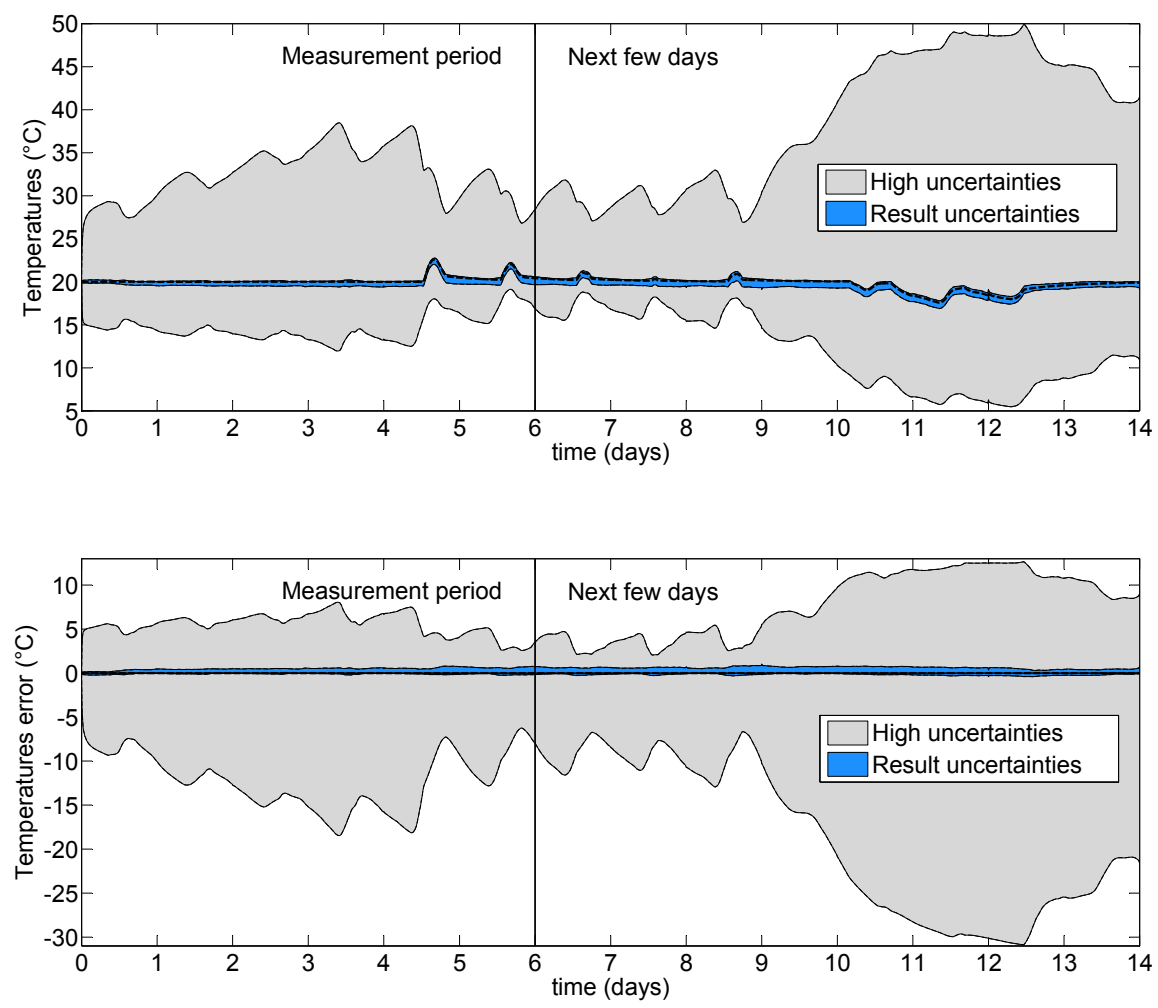

Figure 8: Extreme bounds of thermal response of the identified models for the first zone during the measurement period and the week after. On top, the temperatures ; on bottom, the error graph.

consumption. We can see the large uncertainty band in the highly uncertain case with a $\pm 2000 \mathrm{~W}$ uncertainty, and the reconstructed uncertainty band less than $\pm 200 \mathrm{~W}$.

We then compute the energy flux decomposition in table 12. Here again the previous results are confirmed and the uncertainties are highly reduced. Naturally, these values are higher than the one obtained in table 9 with the classical initial uncertainties.

This study proves the robustness of the method with respect to the initialization of the unknowns. We can conclude that the data gathering's operating procedure can be easily reconsidered in order to focus on the specification of all parameters supposed to be known. 

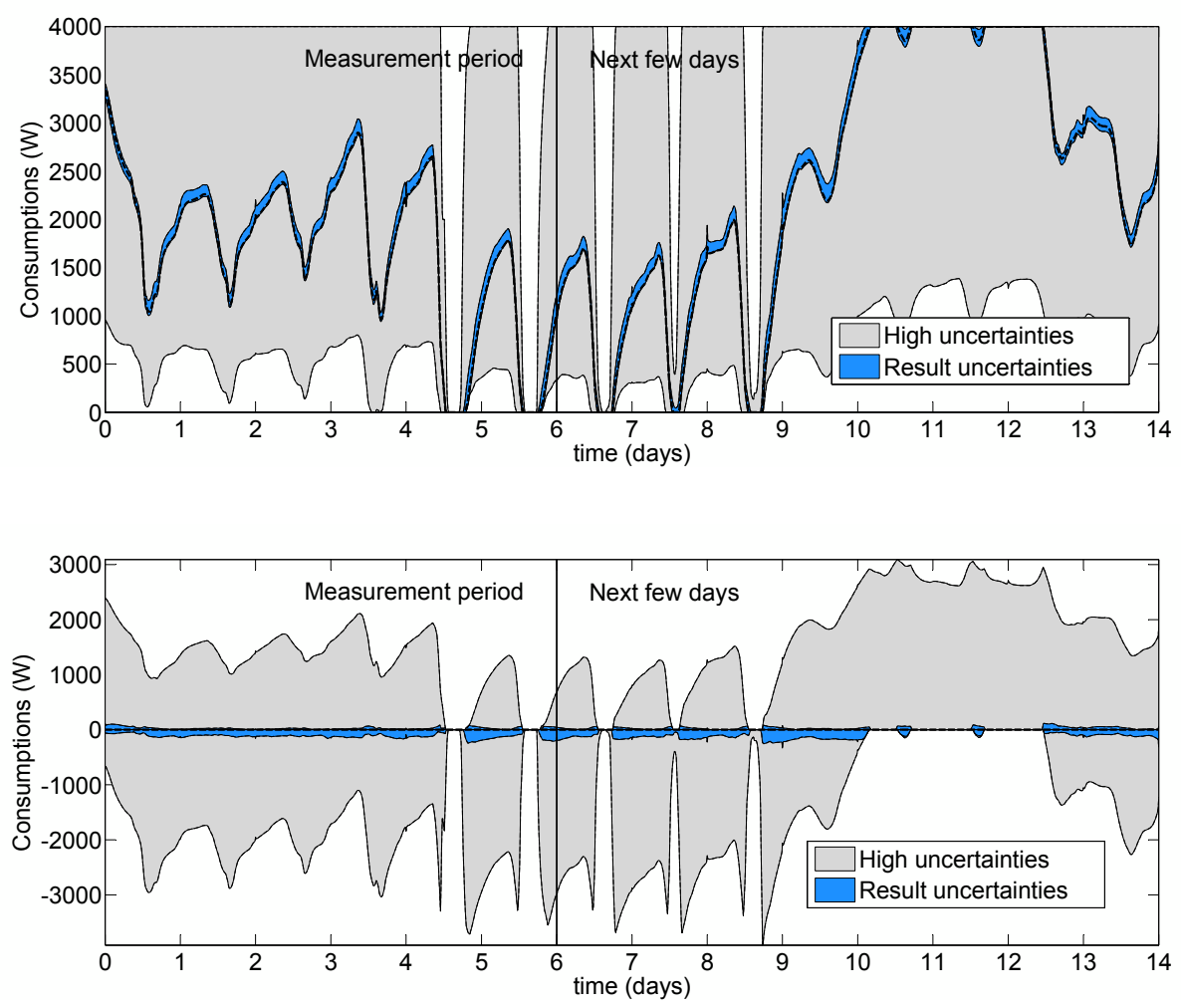

Figure 9: Extreme bounds of the computed intrinsic minimal consumption of the identified models for the first zone during the measurement period and the week after. On top, the power supplied to heating devices ; on bottom, the error graph.

\subsection{Noise level influence on results}

This parametric study evaluates the influence of the measurements noise level on the results of the energy performance assessment method. Again, the numerical experiment takes place in the city of Lyon (France) during the first week of February 2014, and we suppose that we have 6 days of measurements. We add a white Gaussian noise to the thermal state measurements, with a standard deviation evolving in the set $\{0,0.1,0.5,1\}$. We show in figure 10 the noise level for the zone 1 temperature for standard deviation equals to 0.5 and 1. The 0.5 standard deviation is a classical noise level, and the 1 standard deviation represents a high noise level. This noise is added to the thermal state sensors: wall surface temperatures, heating device surface temperatures, and zone air temperatures. We then take back the Monte-Carlo sample of 50 randomly chosen parameters with classical building parameter uncertainties (see section 3.4), and run the audit method as in section 3.4. 


\begin{tabular}{c|c|r|r|r|r} 
Method & Flux $\left(\mathrm{kWh} / \mathrm{m}^{2}\right.$.year $)$ & Target & Value & $\bar{E}_{\%}$ & $\sigma_{\%}$ \\
\hline \multirow{4}{*}{ Highly uncertain case } & $\int \overline{\mathcal{A}}$ & -54.37 & -58.39 & 7.4 & 45 \\
& $\int \mathcal{\mathcal { C } ^ { s }}$ & -496.23 & -456.99 & 7.9 & 19.8 \\
& $\int \mathcal{N}$ & -37.30 & -84.70 & 127 & 269.8 \\
& $\int \Phi$ & 42.20 & 48.63 & 15.3 & 46.9 \\
& $\int \mathcal{W}$ & 335.73 & 294.08 & 12.4 & 29.8 \\
\hline \multirow{3}{*}{ Developed method } & $\int \mathcal{\mathcal { A }}$ & -54.37 & -70.86 & 30.3 & 52 \\
& $\int \mathcal{C}^{s}$ & -496.23 & -492.65 & 0.7 & 2.2 \\
& $\int \mathcal{N}$ & -37.30 & -30.54 & 18.1 & 54.3 \\
& $\int \Phi$ & 42.20 & 46.78 & 10.9 & 13.9 \\
& $\int \mathcal{W}$ & 335.73 & 344.06 & 2.5 & 6
\end{tabular}

Table 12: Target, mean and standard deviation of the energy flux decomposition reconstructions.
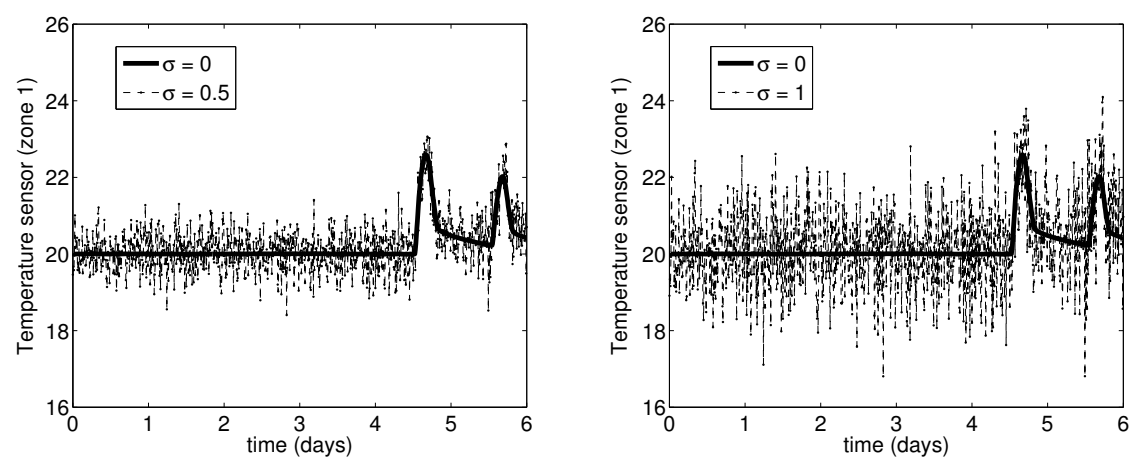

Figure 10: Noise-free and noisy measures of the zone 1 temperature sensor.

We plot in figure 11 the parameters estimator's standard deviation versus the noise level. We can see that the measurement noise does not affect all the parameter estimations in the same way. Indeed, only the identifications of $d_{z}$ (the heating device capacities) seems to be really perturbed by the noise increase, all other estimator standard deviations remain quite constant. This could be explained by the fact that the selected unknowns are composed of intrinsic thermal parameters, which account for the fundamental modes of the thermal response. Since the Gaussian white noise of the thermal response has a zero mean value, the identification process is not highly disrupted by the noise. The result for the reconstruction of $d_{z}$ can be explained by cost-function's lake of thermal sensitivity with respect to this parameter.

We now compute the reconstructed thermal state of the first zone, and compare it to the uncertain one. We plot its extreme bounds in figure 12. We can see that, even in a highly noisy case, the uncertainty of the building thermal state is greatly reduced, going from a $\pm 2^{\circ} \mathrm{C}$ uncertainty to less than $\pm 0.1^{\circ} \mathrm{C}$. 


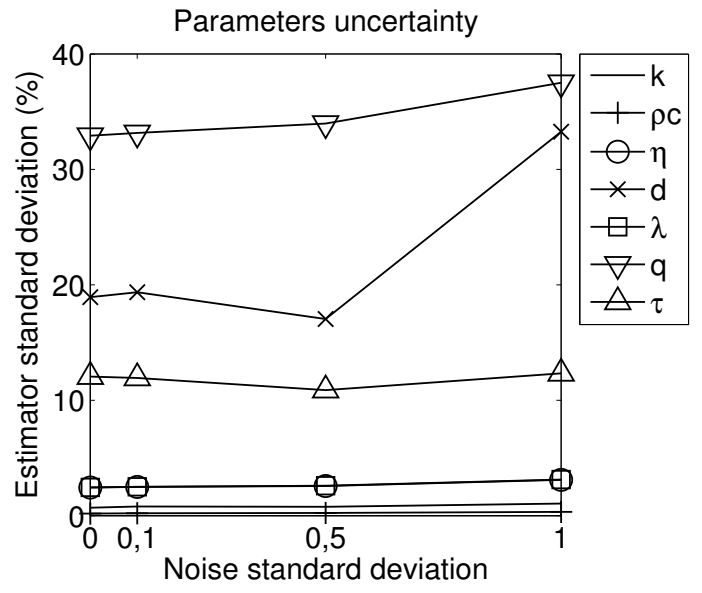

Figure 11: Parameters estimator's standard deviation versus the thermal state sensor's noise level.

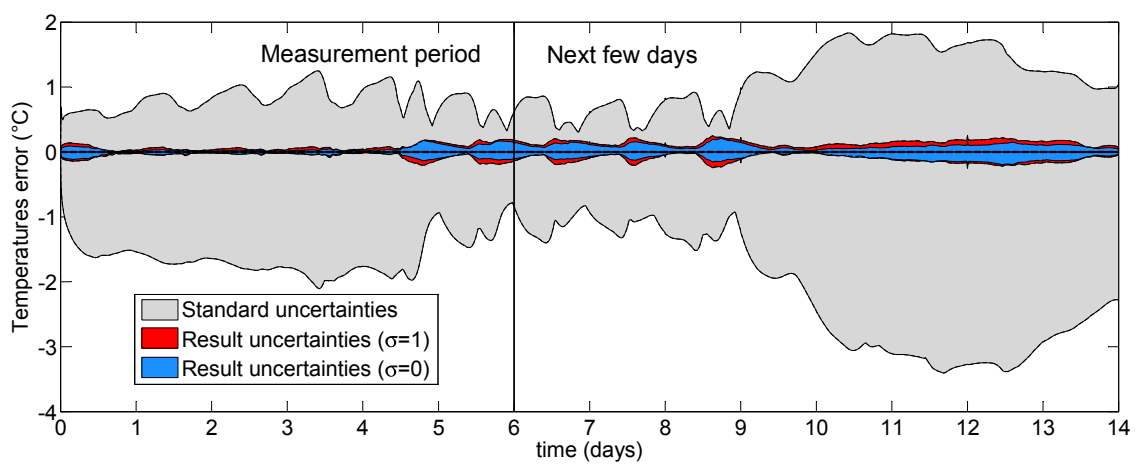

Figure 12: Extreme bounds of thermal response of the identified models for the first zone for different noise level. In red, with $\sigma=1$ standard deviation; in blue, without noise.

We then apply the quantities of interest to the identification results. We show in table 13 and in figure 13 the computation of the minimal intrinsic consumption for the standard uncertain case and for the different noise levels. Even for a 1 standard deviation noise on thermal state measurements, we can see that this quantity is greatly improve, going from a $9 \%$ uncertainty to less than $0.5 \%$. Moreover, the increase of the thermal state measurement noise does not particularly impact the computation of the intrinsic minimal consumption.

We apply the energy flux decomposition analysis to the results, and plot the evolution of the estimator standard deviation with respect to the noise level in figure 14. As for the parameter reconstructions, we can see that the increase of 


\begin{tabular}{l|c|l|l} 
Minimal intrinsic consumption & Value $\left(\mathrm{kWh} / \mathrm{m}^{2}\right.$. year $)$ & $\bar{E}_{\%}$ & $\sigma_{\%}$ \\
\hline Target & 373.05 & - & - \\
Standard method & 367.94 & 1.4 & 9 \\
Developed method $(\sigma=0)$ & 372.93 & 0 & 0.4 \\
Developed method $(\sigma=0.1)$ & 372.91 & 0 & 0.4 \\
Developed method $(\sigma=0.5)$ & 372.91 & 0 & 0.4 \\
Developed method $(\sigma=1)$ & 373.92 & 0 & 0.5
\end{tabular}

Table 13: Time-integration of the minimal intrinsic consumption reconstruction for different noise level.

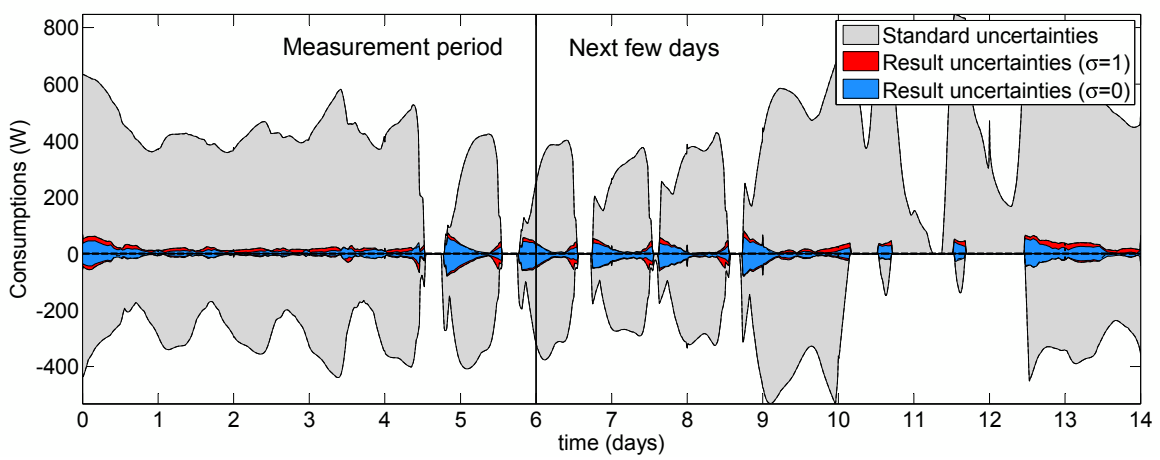

Figure 13: Extreme bounds of the energy consumption of the identified models for the first zone during the measurement period and the week after. In red, with $\sigma=1$ standard deviation; in blue, without noise.

the measurement noise has only a low impact on the energy flux decomposition. That is, the energy performance assessment conclusions in a noise-free case will be similar to those in a noisy case.

This study proves that the energy performance assessment method is quite stable with respect to the noise level of the thermal state measurement. Concretely, it suggests that the method will perform as well with low cost thermal sensors than with high cost ones.

\subsection{Using other weather conditions}

This parametric study evaluates the influence of the season of the thermal diagnosis on the results of the energy performance assessment method. The numerical experiment takes place during the first weeks of February, May, August and November 2014, and we suppose that we have 6 days of measurements. The environmental solicitations are sum up in table 14 in terms of degree day and daily mean solar flux. We use again the Monte-Carlo sample of 50 randomly 


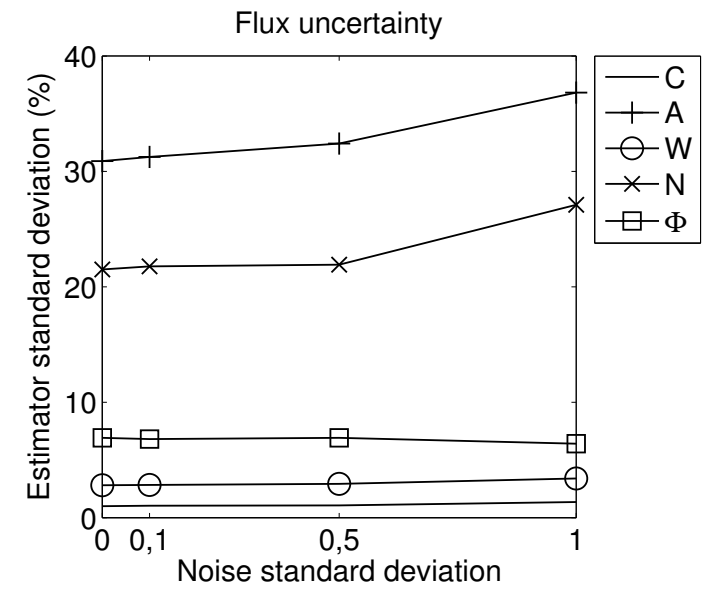

Figure 14: Standard deviation of the energy flux decomposition reconstructions for different noise level on the thermal state measurement .

chosen parameters with classical building parameter uncertainties and run the audit method as in section 3.4.

\begin{tabular}{l|c|c|c}
\multirow{2}{*}{ Month } & Mean degree day $\left({ }^{\circ} \mathrm{C}\right)$ & Mean daily solar flux $(\mathrm{kWh})$ \\
& & Beam & Diffuse \\
\hline February & 12,38 & 2,01 & 0,95 \\
May & 6,15 & 1,88 & 3,08 \\
August & $-2,48$ & 4,73 & 2,78 \\
November & 9,56 & 1,15 & 1,13
\end{tabular}

Table 14: Mean degree day and daily mean solar flux for the different audit periods. The base used to compute the degree days is $20^{\circ} \mathrm{C}$.

We plot in figure 15 the parameters estimator's standard deviation versus the diagnosis month. We can see that the method performed well for all the tested seasons. Still, the parameter estimation appears to be slightly improved in the summer period. This might be related to the behavior of the adjoint method to compute the cost function gradient and identifiability criteria appearing in equations (14)-(20).

We then apply the quantities of interest to the identification results. We show in table 15 the computation of the minimal intrinsic consumption for the standard case and for the different diagnosis months. The results are quite constant along the year.

We apply the energy flux decomposition analysis to the results, and plot the evolution of the estimator standard deviation with respect to the audit month in figure 16. Again, we can see that the diagnosis month only has a low impact on the energy flux decomposition. The method looks to perform as well in summer as in winter periods. 


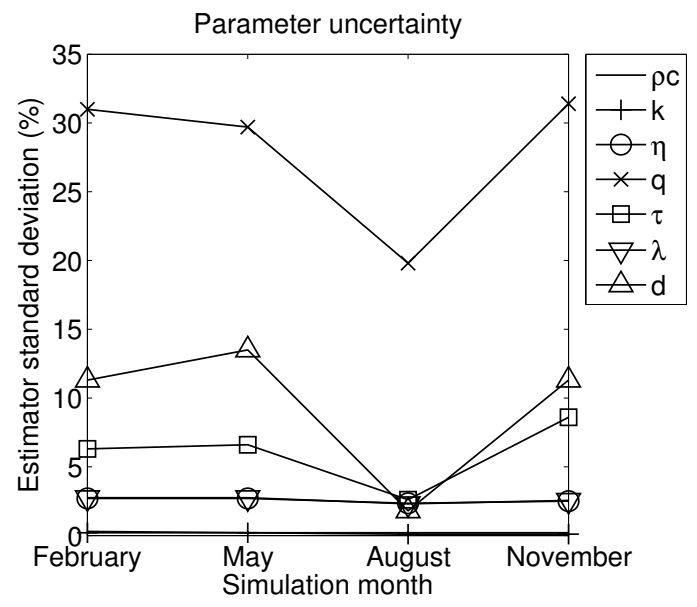

Figure 15: Parameters estimator's standard deviation versus the audit month.

\begin{tabular}{l|c|l|l} 
Minimal intrinsic consumption & Value $\left(\mathrm{kWh} / \mathrm{m}^{2}\right.$. year $)$ & $\bar{E}_{\%}$ & $\sigma_{\%}$ \\
\hline Target & 373.05 & - & - \\
Standard method in February & 367.94 & 1.4 & 9 \\
Developed method in February & 373.05 & 0 & 0.4 \\
Developed method in May & 373.01 & 0 & 0.5 \\
Developed method in August & 372.86 & 0.1 & 0.6 \\
Developed method in November & 373.04 & 0 & 0.4
\end{tabular}

Table 15: Minimal intrinsic consumption obtained with the calibrated model for different seasons.

This study proves that the energy performance assessment method does not strongly depend on the diagnosis weather conditions. We run the method in four different months over the year (one per season) and the results remain almost the same, with a slight improvement in the summer period for the parameter identification.

\subsection{Sensor data generated with EnergyPlus}

The last section introduces a series of more realistic tests. Here the sensors data are generated by a full-detail whole-building model implemented in EnergyPlus. Some outliers appear in the weather data at every midnight time, and thus propagate in the sensors data. Since they appear both in the weather data and the sensors data, they do not perturb the present numerical application. This is why you can observe some outliers in the thermal response and heating system power supplied plots.

Here again, we study the results of the audit method. Unfortunately, EnergyPlus does not provide the heating device thermal state as needed for our tool, and we can't reasonably hope to reconstruct all unknowns without this data. 


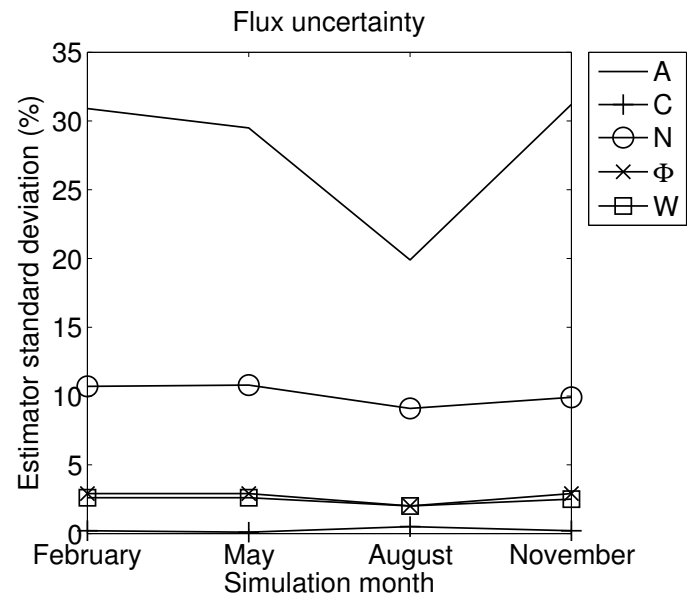

Figure 16: Standard deviation of the energy flux decomposition reconstructions for different seasons.

Thus we reduced the set of unknown parameters and removed the heating devices characteristics:

$$
u=\left\{\rho c_{s}, k_{s}, \bar{q}_{z}, \tau_{z}\right\}_{s, z}
$$

The following results are hence obtained with a reduced inverse model.

Similarly as in 3.4, the numerical experiment takes place in the city of Lyon (France) during the first week of February 2014, and we suppose that we have 6 days of measurements coming from the outputs of the EnergyPlus building thermal simulation. These data are composed of the zone air temperatures and some wall surface temperatures. We take back the Monte-Carlo sample of 50 randomly chosen parameters with classical uncertainties as standard energy diagnosis methods, and use again these values as initialization for our estimation method.

The results are presented in table 16. We can see that the thermal properties of the walls are accurately identified with low mean errors and uncertainties reduced from $20-25 \%$ to less than $2 \%$. These result has already been highlight in the work [25]. The reconstruction of $\bar{q}_{z}$ has only a $10 \%$ uncertainty, whereas $30 \%$ with standard approach. The window transmittance is correctly identified by our estimation method, with less than $3.2 \%$ uncertainties. We can see that the mean errors of the estimated parameters are not null. This is due to the bias introduced by the non-calibration of the heating system characteristics.

We then compute the reconstructed thermal state of the first zone, and compare it to the uncertain one. We plot its extreme bounds in figure 17. We can see that the uncertainty of the building thermal state is greatly reduced, going from $\mathrm{a} \pm 2^{\circ} \mathrm{C}$ uncertainty to less that $\pm 0.2^{\circ} \mathrm{C}$ uncertainty.

We apply the quantities of interest to the identification results. We show in table 17 the minimal intrinsic consumption computation. We can see that the uncertainty of this quantity is greatly improve, at least by a 10 factor. Here 


\begin{tabular}{c|c||c|c|c||c|c|c}
\multirow{2}{*}{ Parameter } & \multicolumn{2}{|c|}{ Target } & \multicolumn{3}{c||}{ Standard method } & \multicolumn{3}{c}{ Developed method } \\
& $u_{\text {tar }}$ & $\bar{u}$ & $\bar{E}_{\%}$ & $\sigma_{\%}$ & $\bar{u}_{r e c}$ & $\bar{E}_{\%}$ & $\sigma_{\%}$ \\
\hline$\rho c_{s}$ & $1.800 \mathrm{e}^{6}$ & $1.713 \mathrm{e}^{6}$ & 4.8 & 21.2 & $1.773 \mathrm{e}^{6}$ & 1.5 & 1.6 \\
$k_{s}$ & 0.960 & 0.910 & 5.2 & 24.3 & 0.949 & 1.1 & 1.8 \\
$\bar{q}_{z}$ & $5.1 \mathrm{e}^{-3}$ & $5.1 \mathrm{e}^{-3}$ & 0.9 & 30.4 & $4.3 \mathrm{e}^{-3}$ & 15 & 9.3 \\
$\tau_{z}$ & 0.50 & 0.49 & 1.2 & 11.3 & 0.20 & 59.8 & 3.2
\end{tabular}

Table 16: Target, mean and standard deviation of reconstructed values with the Monte-Carlo sample with EnergyPlus generated sensor data.
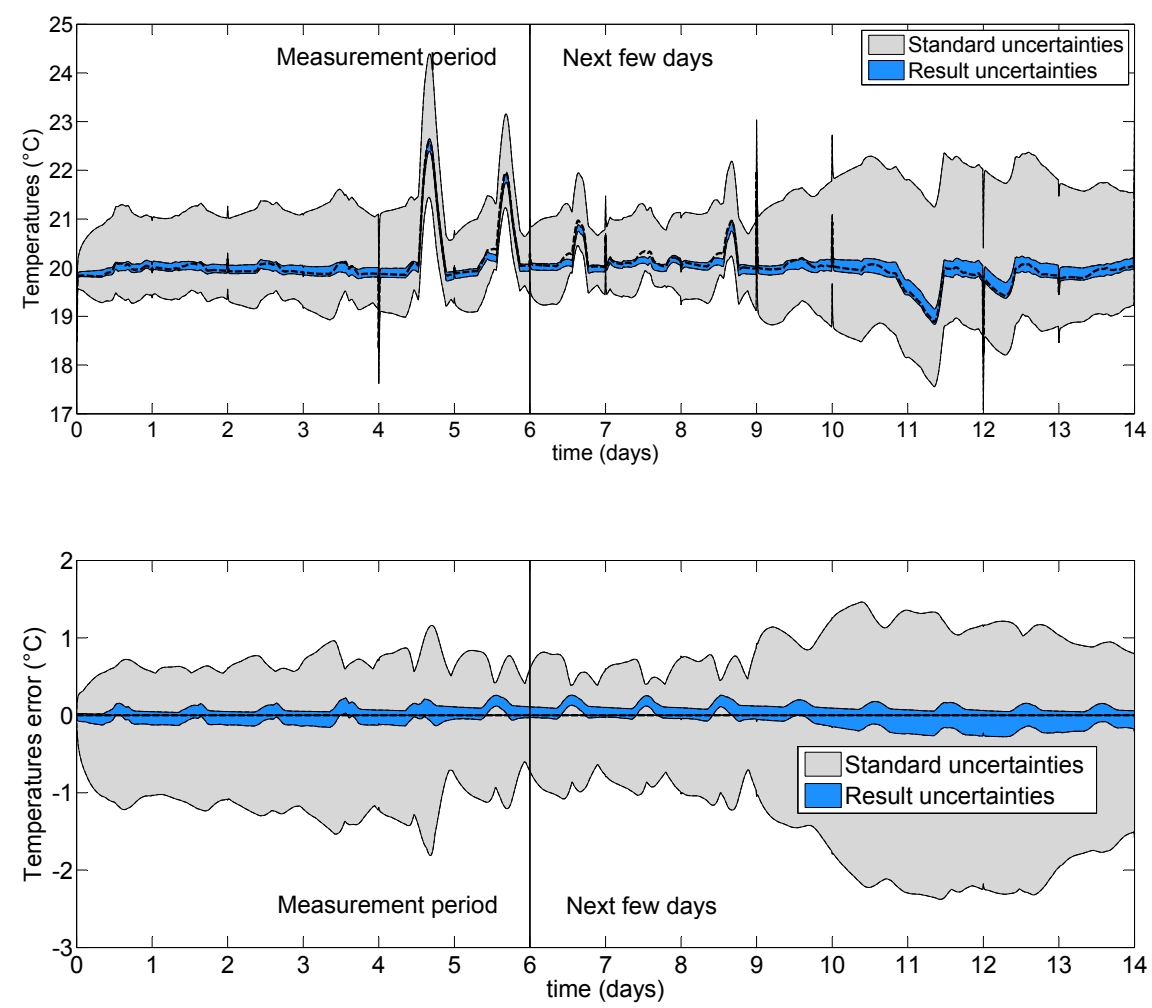

Figure 17: Extreme bounds of thermal response of the identified models for the first zone during the measurement days and some days after. On top, the temperatures ; on bottom, the error.

again, the mean error can be explained by the fact that the heating devices have not been calibrated by our method, so it contains a modeling error which results in a bias.

We plot in figure 18 the extreme bounds of the reconstructed intrinsic min- 


\begin{tabular}{l|c|l|l} 
Minimal intrinsic consumption & Value $\left(\mathrm{kWh} / \mathrm{m}^{2}\right.$. year $)$ & $\bar{E}_{\%}$ & $\sigma_{\%}$ \\
\hline Target & 357.16 & - & - \\
Standard method & 366.40 & 2.6 & 7.9 \\
Developed method & 373.36 & 4.5 & 0.6
\end{tabular}

Table 17: Time-integration of the minimal intrinsic consumption reconstruction for sensors data generated by EnergyPlus software.

imal consumption. We can see the uncertainty band in the standard case is around $\pm 300 \mathrm{~W}$, and the estimated uncertainty band is less than $\pm 50 \mathrm{~W}$. The bias appears here again, since the mean value of the estimated minimal intrinsic consumption error graph is not centered at zero.
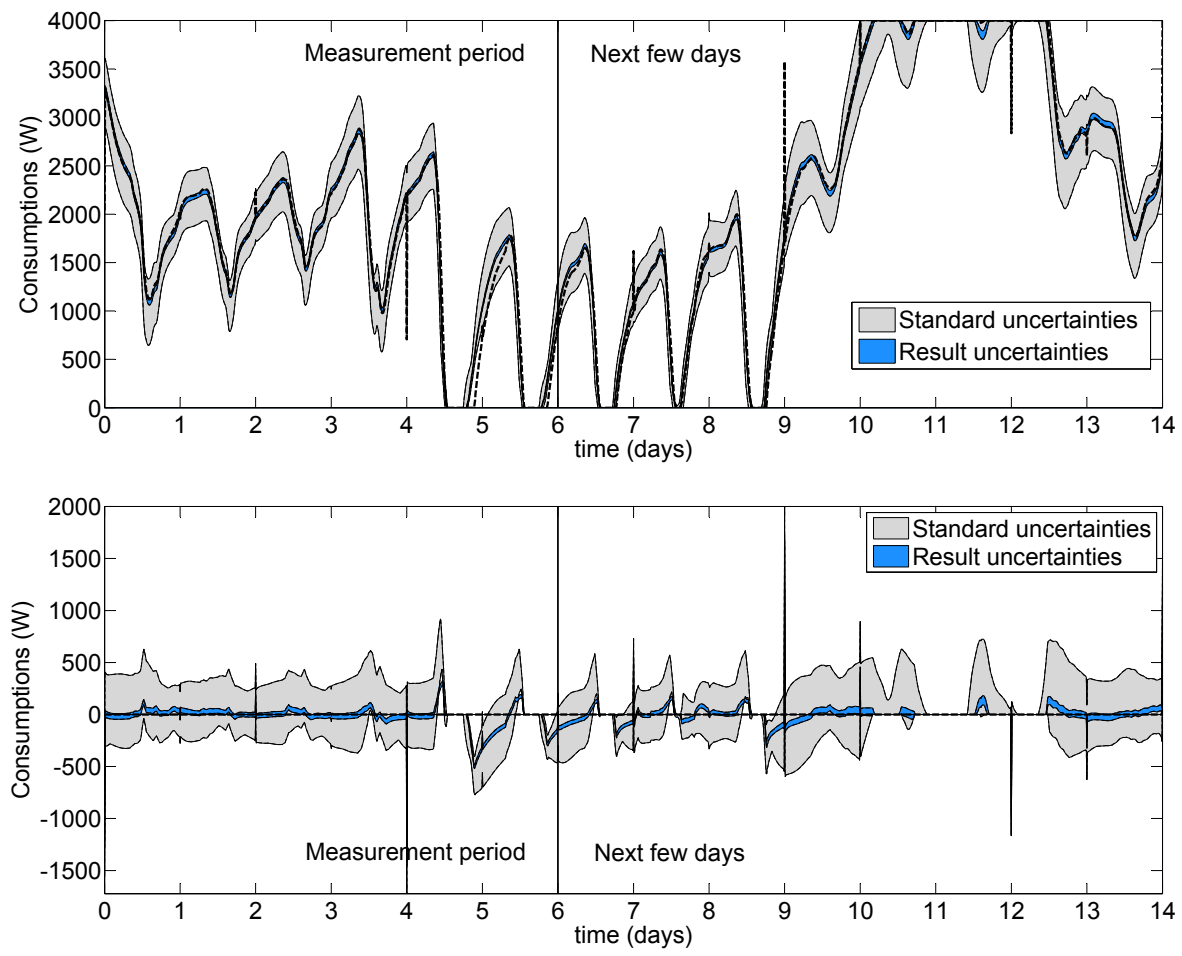

Figure 18: Extreme bounds of computed intrinsic minimal consumption of the identified models during the measurement days and the week after. On top, the consumption ; on bottom, the error. The dotted line corresponds to the EnergyPlus prediction that serves as a reference.

We apply the energy flux decomposition analysis to the results in table 18 . We can conclude that the energy flux decomposition obtained with our method 
has lower uncertainties than standard approaches. The errors in the mean value $\bar{E}_{\%}$ may result from the modeling error introduced by the ill-knowledge of heating device characteristics. However, the reduction of the uncertainties $\sigma_{\%}$ with sensors data coming from an exterior building thermal modeling software is an important result.

\begin{tabular}{c|c|r|r|r|r} 
Method & Flux $\left(\mathrm{kWh} / \mathrm{m}^{2}\right.$. year $)$ & Target & Value & $\bar{E}_{\%}$ & $\sigma_{\%}$ \\
\hline \multirow{3}{*}{ Standard method } & $\int \overline{\mathcal{A}}$ & -53.62 & -53.31 & 0.6 & 28.9 \\
& $\int \mathcal{C}^{s}$ & -483.38 & -482.53 & 0.2 & 3.4 \\
& $\int \Phi$ & 42.20 & 41.69 & 1.2 & 11.3 \\
\hline \multirow{3}{*}{ Developed method } & $\int \overline{\mathcal{A}}$ & -53.62 & -44.60 & 16.8 & 8.1 \\
& $\int \mathcal{C}^{s}$ & -483.38 & -467.89 & 3.2 & 0.7 \\
& $\int \Phi$ & 42.20 & 16.91 & 59.9 & 0.8
\end{tabular}

Table 18: Target, mean and standard deviation of the energy flux decomposition reconstructions.

We have to remember that we had to use here a reduced inverse model. The fact that EnergyPlus does not allow to extract heating device surface temperatures introduces another modeling error on their thermal characteristics. That's exactly why our energy performance assessment method aims at identifying these parameters: to reduce the intrinsic modeling error. The main result of this section is that the quantities of interest have lower uncertainties with our method than with standard approaches.

\section{Evaluation of refurbishment scenarios}

The energy flux decomposition is a decision making tool helping professionals in the choice of a refurbishment scenario. We illustrate this assertion on a numerical example. Let us consider a case where the proposed work is a renovation of the envelope or of the heating systems. We recall the previous uncertainties obtained in section 3.4 for these two energy flux, but we rounded the values for illustration purpose.

\begin{tabular}{c|c|c|c}
\multirow{2}{*}{ Flux } & Value $\left(\mathrm{kWh} / \mathrm{m}^{2}\right.$. year $)$ & \multicolumn{2}{|c}{ Uncertainty $(\sigma \%)$} \\
& & Standard & Developed \\
\hline $\int \mathcal{C}^{s}$ & -500 & $\pm 5 \%$ & $\pm 1 \%$ \\
$\int \mathcal{N}$ & -40 & $\pm 50 \%$ & $\pm 20 \%$
\end{tabular}

Table 19: Target, mean and standard deviation of the energy flux decomposition reconstructions.

For example, suppose that the return of investment for the insulation work is 10 years and 8 years for the changing of heating systems. Following the value of table 19, we obtain the uncertainty of the return of investments presented 
in table 20. As you can see with standard methods, the uncertainty band of the return of investment for the two renovation scenarios are overlapping. With the developed method, the uncertainty band are reduced and didn't overlap anymore.

\begin{tabular}{c|c|c}
\multirow{2}{*}{ Renovation work } & \multicolumn{2}{|c}{ Return of Investment } \\
& Standard & Developed \\
\hline Insulation & 10 years \pm 6 month & 10 years \pm 1 month \\
Heating systems & 8 years \pm 4 years & 8 years \pm 1.5 years
\end{tabular}

Table 20: Computation of the return of investment.

This small example illustrates the use of the energy performance assess method and quantities of interest as an expert tool to help professionals in the choice of refurbishment scenarios. These particular output of the method will be subject to a comprehensive presentation and analysis in future works with application to larger scale building.

\section{Conclusions}

We propose an automated measure-based energy performance assessment method that accurately predicts the overall energy consumption and the energy flux decomposition. It requires thermal measurements, weather data and use conditions to determine some among the most influent intrinsic physical parameters appearing in a detailed building thermal model. This method consists in solving a non linear inverse problem to calibrate the model from the temperature data using the adjoint state technique. We apply this tool to a numerical case study and compare the results with standard energy diagnosis. We show that the developed method highly reduce the standard manual data collection uncertainties. Moreover, we investigate the ability of the identification method to compute the quantities of interest composed by the minimal intrinsic consumption and the energy flux decomposition. They represent crucial information for the evaluation of refurbishment scenarios impact, thus providing with decision making financial parameters that will ultimately help improving return on investment and establishing energy performance contracts. This new energy performance assessment method allows to accurately evaluate the interest variables and thus to improve the energy audit outcomes. Moreover, it affords to propose reliable energy performance contracts.

We study the result sensitivities with respect to the initial uncertainties, the measurement noise, the month of the diagnosis and the modeling error. The study of the high initial uncertainties proves the robustness of the method with respect to the initialization. We also show that the developed method is robust with respect to the season of diagnosis and the measurement noise, since the quantities of interest are accurately computed in all seasons and are slightly disturbed by the measurement noise increase. The last study evaluates the 
behavior of the energy performance assessment method when facing modeling error. To do that, we used a full-detail whole-building model implemented in EnergyPlus software to generate the sensor data. Since this software does not output the heating device surface temperatures, we had to use a reduced inverse model, introducing by the way another modeling error. In this case, the results present a bias, but the uncertainties in the quantities of interest are lower than the ones in the standard approach. We finally provide an use case that illustrates the contribution of the method as an expert tool helping professionals to evaluate refurbishment scenarios and compare return of investments.

The use of a more detailed building model, with air and moisture balance and HVAC, may increase the scope of the method and probably ease the real case application. Indeed, the nodal multizone building model supports heat, air and moisture (HAM) transfer using analogous equations than the thermal ones. This observation suggests that the inverse formulation introduced here could easily be extended to provide HAM state variables and identify these

fields simultaneously with the use of additional relative humidity and air mass concentration sensors. It may also improve the air renewal estimation, about which the cost function seems to suffer from a thermal sensitivity lack.

\section{Acknowledgments}

This work has been supported by the French Research Funding Agency (ANR) as part of program HABISOL (project MEMOIRE number ANR-10HABISOL-006).

\section{References}

[1] Abushakra, B., 1997. An inverse model to predict and evaluate the energy performance of large commercial and institutional buildings. In: Building Simulation.

[2] Antonopoulos, K. A., Koronaki, E. P., 2000. Effect of indoor mass on the time constant and thermal delay of buildings. International Journal of Energy Research 24, 391-402.

[3] Beck, J. V., Arnold, K. J., 1977. Parameter estimation in engineering and science. John Wiley and sons.

[4] Boland, J., 1997. Simplifying the solution of the differential equations which describe heat flows in domestic dwellings. Building and Environment 32, 479-484.

[5] Bordass, B., Cohen, R., Field, J., 2004. Energy performance of nondomestic buidings. Closing the credibility gap. In: Building Performance Congress.

[6] Boyer, F., Hubert, F., 2014. Méthodes de décomposition de domaine de type Schwarz. 
[7] Brezis, H., 2008. Analyse fonctionnelle. Dunod.

[8] Brouns, J., 2014. Développement d'outils numériques pour l'audit énergétique des bâtiments. Ph.D. thesis, Université Paris-Est.

[9] Carslaw, H. S., Jaeger, J. C., 1959. Conduction of heat in solids. Oxford.

[10] Chavent, G., 2009. Nonlinear least squares for inverse problems. Theoretical foundations and step-by-step guide for applications. Springer-Verlag.

[11] Ciarlet, P. G., 2001. Introduction to numerical linear algebra and optimisation.

[12] Demanuele, C., Tweddell, T., Davies, M., 2010. Bridging the gap between predicted and actual energy performance in schools. In: World Renewable Energy Congress XI.

[13] Engl, H. W., Hanke, M., Neubauer, A., 1996. Regularization of inverse problems. Kluwer Academic.

[14] Gautier, A., Charles, T., 2012. Analyse de sensibilité et d'incertitudes. Tech. rep., SOLAMEN, projet MEMOIRE.

[15] Ghiaus, C., 2006. Experimental estimation of building energy performance by robust regression. Energy and Buildings $38,582-587$.

[16] Güçyeter, B., Günaydın, H. M., 2012. Optimization of an envelope retrofit strategy for an existing office building. Energy and Buildings 55, 647-659.

[17] Judkoff, R. D., Neymark, J. S., 1995. Building Energy Simulation Test (BESTest) and diagnostic method. Tech. rep., National Renewable Energy Laborator.

[18] Karatasou, S., Santamouris, M., Geros, V., 2006. Modeling and predicting building's energy use with artificial neural networks: Methods and results 38, 949-958.

[19] Kuate, R., Nassiopoulos, A., Bourquin, F., 2014. A vectorial descent stepsize for parameter identification of a coupled parabolic PDE-ODE. Inverse Problems in Science and Engineering.

[20] Kunisch, K., 1988. A review of some recent results on the output least squares formulation of parameter estimation problems. Automatica 24(4), $531-539$.

[21] Lions, J. L., 1971. Optimal control of systems governed by partial differential equations. Springer-Verlag.

[22] Macdonald, I. A., 2002. Quantifying the effect of uncertainty in building simulation. Ph.D. thesis, University of Strathclyde, UK. 
[23] Mejri, O., Palomo Del Barrio, E., Ghrab-Morcos, N., 2011. Energy performance assessment of occupied buildings using model identification techniques. Energy and Buildings 43, 285-299.

[24] Moré, J. J., 1977. The Levenberg-Marquardt algorithm. Implementation and theory. In: Conference on Numerical Analysis.

[25] Nassiopoulos, A., Bourquin, F., 2013. On-site building walls characterization. Numerical Heat Transfer 63, 1-22.

[26] Nassiopoulos, A., Brouns, J., Artiges, N., Smail, M.-K., Azerou, B., 2014. ReTrofiT: A software to solve optimization and identification problems applied to building energy management. In: EWSHM - 7th European Workshop on Structural Health Monitoring.

[27] Norford, L. K., Socolow, R. H., Hsieh, E. S., Spadaro, G. V., 1994. Twoto-one discrepancy between measured and predicted performance of a lowenergy office building. Insights from a reconciliation based on the DOE-2 model. Energy and Buildings 21, 121-131.

[28] Pan, Y., Huang, Z., Wu, G., 2007. Calibrated building energy simulation and its application in a high-rise commercial building in Shanghai. Energy and Buildings 39, 651-657.

[29] Pedrini, A., Westphal, F. S., Lamberts, R., 2002. A methodology for building energy modelling and calibration in warm climates. Building and Environment $37,903-912$.

[30] Rabl, A., 1988. Parameter estimation in buidings. Methods for dynamic analysis of measured energy use. Solar Energy Engineering 110, 52-56.

[31] Raviart, P.-A., Thomas, J.-M., 1998. Introduction à l'analyse numérique des équations aux dérivées partielles. Dunod.

[32] Raynaud, M., 2014. évaluation ex-post de l'efficacité de solutions de rénovation énergtique en résidentiel. Ph.D. thesis, École nationale supérieure des mines de Paris.

[33] Spitz, C., 2012. Analyse de la fiabilité des outils de simulation et des incertitudes de métrologie appliquée à l'efficacité énergétique des bâtiments. Ph.D. thesis, Université de Grenoble.

[34] Tarantola, A., 2005. Inverse problem theory and methods for model parameter estimation. SIAM.

[35] Tikhonov, A. N., Arsenin, V. Y., 1977. Solutions of ill-posed problems. Winston.

[36] Tsilingiris, P., 2002. On the transient thermal behaviour of structural walls - the combined effect of time varying solar radiation and ambient temperature. Renewable Energy 27, 319-336. 
[37] Zhao, H.-X., Magoulès, F., 2012. A review on the prediction of building energy consumption. Renewable and Sustainable Energy Reviews 16, 35863592 . 